\title{
Hyperfine spectroscopy in a quantum-limited spectrometer
}

\author{
Sebastian Probst ${ }^{1}$, Gengli Zhang ${ }^{2}$, Miloš Rančić ${ }^{1}$, Vishal Ranjan ${ }^{1}$, Marianne Le Dantec ${ }^{1}$, \\ Zhonghan Zhang ${ }^{3}$, Bartolo Albanese ${ }^{1}$, Andrin Doll ${ }^{4}$, Ren Bao Liu ${ }^{2}$, John Morton $^{5}$, Thierry Chanelière ${ }^{6}$, \\ Philippe Goldner ${ }^{3}$, Denis Vion ${ }^{1}$, Daniel Esteve ${ }^{1}$, and Patrice Bertet ${ }^{1}$ \\ ${ }^{1}$ Quantronics group, SPEC, CEA, CNRS, Université Paris-Saclay, CEA Saclay, \\ 91191 Gif-sur-Yvette CEDEX, France \\ ${ }^{2}$ Department of Physics and The Hong Kong Institute of Quantum Information Science and Technology, \\ The Chinese University of Hong Kong, Shatin, New Territories, Hong Kong, China \\ ${ }^{3}$ Chimie ParisTech, PSL University, CNRS, Institut de Recherche de Chimie Paris, 75005 Paris, France \\ ${ }^{4}$ Laboratory of nanomagnetism and oxides, SPEC, CEA, CNRS, Université Paris-Saclay, CEA Saclay, \\ 91191 Gif-sur-Yvette CEDEX, France \\ ${ }^{5}$ London Centre for Nanotechnology, University College London, London WC1H 0AH, UK \\ ${ }^{6}$ Univ. Grenoble Alpes, CNRS, Grenoble INP, Institut Néel, 38000 Grenoble, France
}

Correspondence: Patrice Bertet (patrice.bertet@cea.fr)

\author{
Received: 21 April 2020 - Discussion started: 22 July 2020 \\ Revised: 25 September 2020 - Accepted: 29 October 2020 - Published: 17 December 2020
}

\begin{abstract}
We report measurements of electron-spin-echo envelope modulation (ESEEM) performed at millikelvin temperatures in a custom-built high-sensitivity spectrometer based on superconducting micro-resonators. The high quality factor and small mode volume (down to $0.2 \mathrm{pL}$ ) of the resonator allow us to probe a small number of spins, down to $5 \times 10^{2}$. We measure two-pulse ESEEM on two systems: erbium ions coupled to ${ }^{183} \mathrm{~W}$ nuclei in a natural-abundance $\mathrm{CaWO}_{4}$ crystal and bismuth donors coupled to residual ${ }^{29} \mathrm{Si}$ nuclei in a silicon substrate that was isotopically enriched in the ${ }^{28} \mathrm{Si}$ isotope. We also measure three- and five-pulse ESEEM for the bismuth donors in silicon. Quantitative agreement is obtained for both the hyperfine coupling strength of proximal nuclei and the nuclear-spin concentration.
\end{abstract}

\section{Introduction}

Electron paramagnetic resonance (EPR) spectroscopy provides a set of versatile tools to study the magnetic environment of unpaired electron spins (Schweiger and Jeschke, 2001). EPR spectrometers rely on the inductive detection of the spin signal by a three-dimensional microwave resonator tuned to the spin Larmor frequency. While concentration sensitivity is the main concern for dilute samples available in macroscopic volumes (Song et al., 2016), there are also cases in which the absolute spin detection sensitivity matters, motivating research towards alternative detection methods to measure smaller and smaller numbers of spins. Electrical (Elzerman et al., 2004; Veldhorst et al., 2014; Morello et al., 2010; Pla et al., 2012), optical (Wrachtrup et al., 1993;
Jelezko et al., 2004), and scanning-probe-based (Rugar et al., 2004; Baumann et al., 2015) detection methods of magnetic resonance have reached sufficient sensitivity to detect individual electron spins.

In parallel, recent results have shown that the inductive detection method can also be pushed to much higher absolute sensitivity than previously achieved, using planar microresonators (Narkowicz et al., 2008; Artzi et al., 2015) and micro-helices (Sidabras et al., 2019). Superconducting resonators (Wallace and Silsbee, 1991; Benningshof et al., 2013; Sigillito et al., 2014) are particularly useful in that context since they combine low mode volume and narrow linewidth $\kappa$. Inductive-detection spectrometers relying on a superconducting planar micro-resonator combined with a 
Josephson parametric amplifier (JPA), cooled down to millikelvin temperatures (Bienfait et al., 2015; Eichler et al., 2017; Probst et al., 2017), have achieved a sensitivity of $10 \mathrm{spin} / \sqrt{\mathrm{Hz}}$ for detecting Hahn echoes emitted by donors in silicon (Ranjan et al., 2020b). A particular feature of these quantum-limited spectrometers is that quantum fluctuations of the microwave field play an important role. First, the system output noise is governed by these quantum fluctuations, with negligible thermal noise contribution. Second, quantum fluctuations also impact spin dynamics by triggering spontaneous emission of microwave photons at a rate $\Gamma_{\mathrm{P}}=4 g^{2} / \kappa$, $g$ being the spin-photon coupling (Bienfait et al., 2016; Eichler et al., 2017; Ranjan et al., 2020a). This Purcell effect forbids $T_{1}$ from becoming prohibitively long since it is at most equal to $\Gamma_{\mathrm{P}}^{-1}$, making spin detection with a reasonable repetition rate possible even at the lowest temperatures.

Hahn echoes are the simplest pulse sequence used in EPR spectroscopy, useful to determine the electron-spin density as well as the spin Hamiltonian parameters and their distribution. The richness of EPR comes from the ability to characterize the local magnetic environment of the electron spins, often consisting of a set of nuclear spins or of other electron spins. For that, hyperfine spectroscopy is required, which uses more elaborate pulse sequences and requires larger detection bandwidth. Previous hyperfine spectroscopy measurements with superconducting micro-resonators include the electron-nuclear double resonance detection of donors in silicon (Sigillito et al., 2017) and the electron-spin-echo envelope modulation (ESEEM) of erbium ions by the nuclear spin of yttrium in a $\mathrm{Y}_{2} \mathrm{SiO}_{5}$ crystal (Probst et al., 2015).

Here, we demonstrate that hyperfine spectroscopy is compatible with quantum-limited EPR spectroscopy despite its additional requirements in terms of pulse complexity and bandwidth, by measuring ESEEM in two model electronspin systems. We measure the ESEEM of erbium ions coupled to ${ }^{183} \mathrm{~W}$ nuclei in a scheelite crystal $\left(\mathrm{CaWO}_{4}\right)$ with a simple two-pulse sequence and get quantitative agreement with a simple dipolar interaction model. We also measure the ESEEM of bismuth donors in silicon caused by ${ }^{29} \mathrm{Si}$ nuclei using two-, three-, and five-pulse sequences (Schweiger and Jeschke, 2001; Kasumaj and Stoll, 2008). Compared to other ESEEM measurements on donors in silicon (Witzel et al., 2007; Abe et al., 2010), ours are performed in an isotopically purified sample having a 100 times lower concentration in ${ }^{29} \mathrm{Si}(500 \mathrm{ppm})$ than natural abundance. As a result, the dominant hyperfine interactions in the ESEEM signal are very low (on the order of $100 \mathrm{~Hz}$ ) and have to be detected at low magnetic fields (around $0.1 \mathrm{mT}$ ). These results bring quantum-limited EPR spectroscopy one step closer to realworld applications. (a)

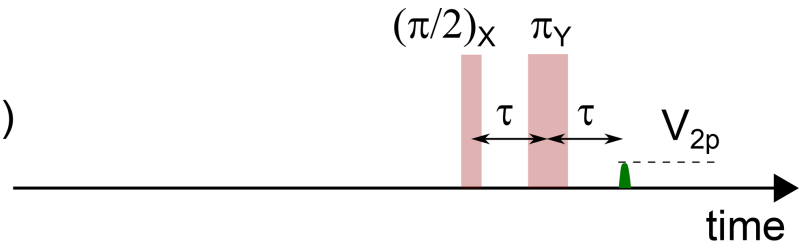

(b)
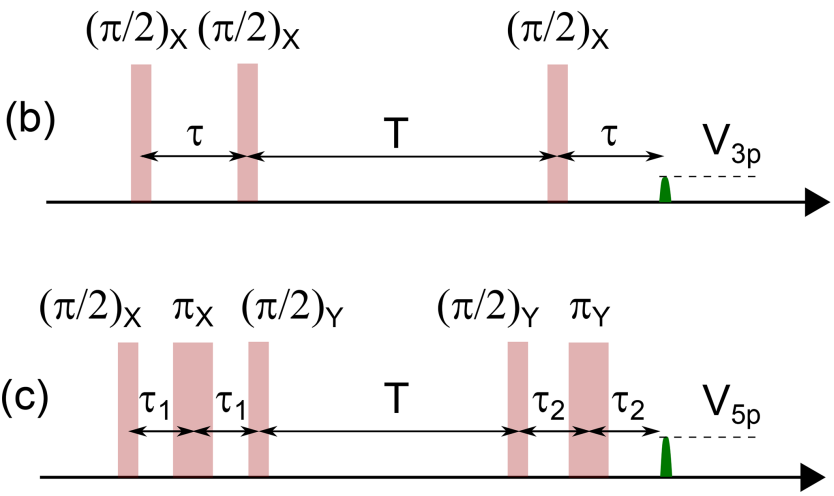

Figure 1. Sequences used for two-pulse (a), three-pulse (b), and five-pulse (c) ESEEM measurements.

\section{ESEEM spectroscopy: theory}

\subsection{Phenomenology}

We start by briefly discussing the ESEEM phenomenon. Consider an ensemble of electron spins placed in a magnetic field $B_{0}$. The spin ensemble linewidth $\Gamma$ is broadened by a variety of mechanisms: spatial inhomogeneity of the applied field $B_{0}$, local magnetic fields generated by magnetic impurities throughout the sample, and spatially inhomogeneous strain or electric fields. One prominent way to mitigate the effect of this inhomogeneous broadening is the spin-echo sequence (also called Hahn echo, or two-pulse echo). It consists of a $\pi / 2$ pulse at time $t=0$ and a $\pi$ pulse after a delay $\tau$ (see Fig. 1a). This $\pi$ pulse reverses the evolution of the phase of the precessing magnetic dipoles, which leads at a later time $2 \tau$ to their refocusing and the emission of a microwave pulse (the echo) of amplitude $V_{2 \mathrm{p}}(\tau)$.

In general, $V_{2 \mathrm{p}}(\tau)$ decays monotonically; it can however also display oscillations. Such ESEEM was first observed by Mims et al. (1961) and Rowan et al. (1965) for $\mathrm{Ce}^{3+}$ ions in a $\mathrm{CaWO}_{4}$ crystal and was interpreted as being caused by the dipolar interaction of the electronic spin of the $\mathrm{Ce}^{3+}$ ions with the ${ }^{183} \mathrm{~W}$ nuclear spins of the crystal. The oscillation frequencies appearing in the ESEEM pattern are related to the nuclear-spin Larmor frequencies and to their coupling to the electron spin. As such, ESEEM measurements provide spectroscopic information on the nature of the nuclear-spin bath and its density, and ESEEM spectroscopy has become an essential tool in advanced EPR (Schweiger and Jeschke, 2001; Mims et al., 1990). ESEEM has also been observed for individual spins measured optically, in particular for individual nitrogen vacancy $(\mathrm{NV})$ centers in diamond coupled to a 
bath of ${ }^{13} \mathrm{C}$ nuclear spins (Childress et al., 2006). A more complete theory of ESEEM is presented in Mims (1972). Our goal here is to provide a simple picture of the physics involved, as well as to introduce useful formulas and notations.

\subsection{Two-spin $1 / 2$ model}

We follow the analysis in Schweiger and Jeschke (2001) of the model case depicted in Fig. 2a. An electron spin of $S=1 / 2$, with an isotropic $g$ tensor, is coupled to a proximal nuclear spin of $I=1 / 2$. Both are subject to a magnetic field $B_{0}$ applied along $z$. The system Hamiltonian is

$\mathbf{H}_{0}=\mathbf{H}_{\mathrm{e}}+\mathbf{H}_{\mathrm{n}}+\mathbf{H}_{\mathrm{hf}}$,

where $\mathbf{H}_{\mathrm{e}}=\omega_{S} \mathbf{S}_{z}\left(\mathbf{H}_{\mathrm{n}}=\omega_{I} \mathbf{I}_{z}\right)$ is the Zeeman Hamiltonian of the electron (nuclear) spin with Larmor frequency $\omega_{S}$ $\left(\omega_{I}\right)$, and $\mathbf{H}_{\mathrm{hf}}$ is the electron-nuclear hyperfine interaction, which includes their dipole-dipole coupling and may include a Fermi contact term as well. We assume that $\omega_{S}$ is much larger than the hyperfine interaction strength, in which case terms proportional to the $\mathbf{S}_{x}$ and $\mathbf{S}_{y}$ operators can be neglected. This secular approximation leads to a hyperfine Hamiltonian of the form $\mathbf{H}_{\mathrm{hf}}=A \mathbf{S}_{z} \mathbf{I}_{z}+B \mathbf{S}_{z} \mathbf{I}_{x}$, with the expressions for $A$ and $B$ depending on the details of the hyperfine interaction (Schweiger and Jeschke, 2001).

Overall, the system Hamiltonian is

$\mathbf{H}_{0}=\omega_{S} \mathbf{S}_{z}+\omega_{I} \mathbf{I}_{z}+A \mathbf{S}_{z} \mathbf{I}_{z}+B \mathbf{S}_{z} \mathbf{I}_{x}$

Because of the $B \mathbf{S}_{z} \mathbf{I}_{x}$ term, the nuclear spin is subjected to an effective magnetic field whose direction (and magnitude) depend on the electron-spin state $\left|\uparrow_{e}\right\rangle$ or $\left|\downarrow_{e}\right\rangle$. Its eigenstates therefore depend on the electron-spin state, so that transitions become allowed between all the spin system energy levels $|1\rangle-|4\rangle$, leading to the ESEEM phenomenon. Relevant parameters are the electron-spin-state-dependent angles between the effective magnetic field seen by the nuclear spin and the quantization axis $z$ :

$$
\begin{aligned}
& \eta_{\uparrow}=\arctan \frac{B}{A+2 \omega_{I}} \\
& \eta_{\downarrow}=\arctan \frac{B}{A-2 \omega_{I}},
\end{aligned}
$$

and the electron-spin-dependent nuclear-spin frequencies:

$$
\begin{aligned}
& \omega_{\uparrow}=\left(\omega_{I}+\frac{A}{2}\right) \cos \eta_{\uparrow}-\frac{B}{2} \sin \eta_{\uparrow} \\
& \omega_{\downarrow}=\left(\omega_{I}-\frac{A}{2}\right) \cos \eta_{\downarrow}-\frac{B}{2} \sin \eta_{\downarrow} .
\end{aligned}
$$

When $\eta_{\uparrow}, \eta_{\downarrow}$ are close to equal, only the nuclear-spinpreserving transitions are allowed; this occurs either when

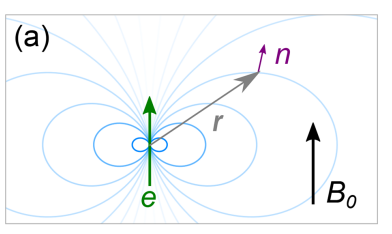

(b)

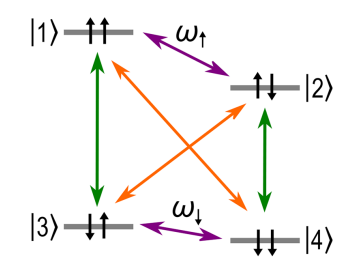

(c)

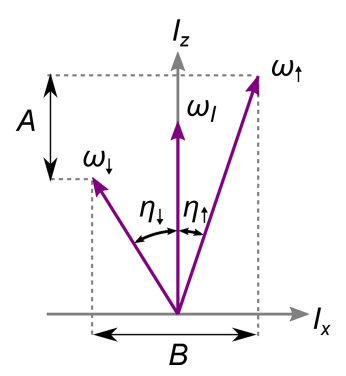

(d)

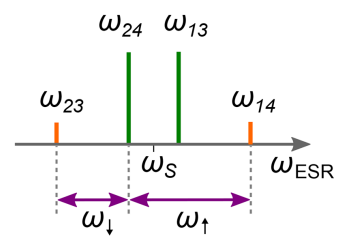

Figure 2. ESEEM model system for an electron spin of $S=1 / 2$ and nuclear spin of $I=1 / 2$ with $\omega_{I}, A, B>0$. (a) Nuclear spin (purple) subject to external field $B_{0}$ and dipole field (blue) of a nearby electron spin (green) located at relative position $\boldsymbol{r}$. (b) Energy diagram showing the electron transitions (green), the nuclear transitions (purple), and the (normally forbidden) electro-nuclear transitions (orange). The energy levels $|1\rangle, \ldots,|4\rangle$ are labeled according to the eigenstates of the Zeeman basis. (c) Quantization axes $\omega_{\uparrow}$ and $\omega_{\downarrow}$ due to mixing of the nuclear states, which results in inclination of the quantization axis from $z$ by the angles $\eta_{\uparrow}$ and $\eta_{\downarrow}$, respectively. (d) EPR spectrum showing the electron transitions (green) and the electro-nuclear transitions (orange) as well as the relation of these electron spin resonance (ESR) transitions to the nuclear frequencies $\omega_{\uparrow}$ and $\omega_{\downarrow}$ (purple).

$B=0$ (due to a specific orientation of the dipolar field or a purely isotropic hyperfine coupling), or when $B \neq 0$ but $\omega_{I} \gg A$ (very weak-coupling limit) or $\omega_{I} \ll A$ (very strong coupling limit). On the contrary, when the direction of the effective magnetic field seen by the nuclear spin is electronspin dependent, all transitions become allowed. This occurs when $B \neq 0$ and $\omega_{I} \simeq \pm A / 2$.

\subsection{Multi-pulse ESEEM}

Because of the level structure shown in Fig. 2, and assuming for simplicity microwave pulses so short that their bandwidth is much larger than $\omega_{\uparrow, \downarrow}$, microwave pulses at the electronspin frequency $\omega_{S}$ excite the allowed transitions $|1\rangle \leftrightarrow|3\rangle$ and $|2\rangle \leftrightarrow|4\rangle$ but also the normally forbidden $|1\rangle \leftrightarrow|4\rangle$ and $|2\rangle \leftrightarrow|3\rangle$, leading to coherence transfer between the levels and to beatings. Note that for simplicity we assume that the microwave pulses are ideal and so short that their bandwidth is much larger than $\omega_{12}$ and $\omega_{34}$.

It is then possible to compute analytically the effect of a two-pulse echo sequence consisting of an instantaneous ideal $\pi / 2$ pulse and an instantaneous ideal $\pi$ pulse (see Fig. 1), disregarding any decoherence. The resulting echo amplitude 
(Schweiger and Jeschke, 2001) is given by

$$
\begin{aligned}
V_{2 \mathrm{p}}(\tau) & =1-\frac{k}{4}\left[2-2 \cos \left(\omega_{\uparrow} \tau\right)-2 \cos \left(\omega_{\downarrow} \tau\right)\right. \\
& \left.+\cos \left(\left(\omega_{\uparrow}-\omega_{\downarrow}\right) \tau\right)+\cos \left(\left(\omega_{\uparrow}+\omega_{\downarrow}\right) \tau\right)\right],
\end{aligned}
$$

with

$k=\left[\frac{B \omega_{I}}{\omega_{\uparrow} \omega_{\downarrow}}\right]^{2}$.

The spin-echo amplitude is modulated by a function whose frequency spectrum and amplitude contain information about the nuclear-spin Larmor frequency $\omega_{I}$ as well as its hyperfine coupling $(A, B)$ to the electron spin. The modulation contrast $0 \leq k \leq 1$ is maximal when transitions $|1\rangle-|4\rangle$ and $|2\rangle-|3\rangle$ are maximally allowed, corresponding to $\omega_{I} \simeq A / 2$.

The above results are exact, as long as the secular approximation is valid and the pulses are ideal. In the weakcoupling limit $A, B \ll \omega_{I}, \omega_{\uparrow} \simeq \omega_{\downarrow} \simeq \omega_{I}$ so that $V_{2 \mathrm{p}}(\tau)=$ $1-\frac{k}{4}\left[3-4 \cos \left(\omega_{I} \tau\right)+\cos \left(2 \omega_{I} \tau\right)\right]$, with $k=\left(B / \omega_{I}\right)^{2} \ll 1$. In this limit, the echo modulation spectrum directly yields the nuclear-spin Larmor frequency and also contains components at twice this frequency. Note however that, in practice, the $\pi$ pulse bandwidth is always finite, because of the resonator bandwidth or limited pulse power; this sets a limit to the range of detectable modulation frequencies.

The electron spin is often coupled to $N$ nuclear spins, with $N>1$. Since all nuclear-spin subspaces can be diagonalized separately, the total ESEEM modulation is simply given by the product of each nuclear-spin modulation $V_{2 \mathrm{p}, l}(\tau), l$ being the nuclear-spin index. Taking also into account that the electron spin is also subject to decoherence processes, modeled, for instance, by an exponential decay with time constant $T_{2}$, the echo envelope is

$V_{2 \mathrm{p}}^{\prime}(\tau)=\exp \left(-2 \tau / T_{2}\right) \prod_{l=1}^{N} V_{2 \mathrm{p}, l}(\tau)$.

The modulation pattern $V_{2 \mathrm{p}}^{\prime}(\tau)$ yields quantitative information about the nature and coupling of the nuclear spins surrounding the electron spin whose echo is measured and is therefore a useful tool in EPR spectroscopy. When the environmental nuclei have a certain probability $p$ to be of a given isotope with a nuclear spin of $I=1 / 2$, and a probability $1-p$ to be of an isotope with $I=0$, the above formulas are straightforwardly modified (Rowan et al., 1965) by writing

$$
\begin{aligned}
V_{2 \mathrm{p}, l}(\tau) & =1-\frac{p k_{l}}{4}\left[2-2 \cos \left(\omega_{\uparrow, l} \tau\right)-2 \cos \left(\omega_{\downarrow, l} \tau\right)\right. \\
& \left.+\cos \left(\left(\omega_{\uparrow, l}-\omega_{\downarrow, l}\right) \tau\right)+\cos \left(\left(\omega_{\uparrow, l}+\omega_{\downarrow, l}\right) \tau\right)\right] .
\end{aligned}
$$

The echo signal $V_{2 \mathrm{p}}^{\prime}(\tau)$ is the sum of terms that have the general form $p^{L} \prod_{l=1}^{l=L} k_{l} \cos \left(\omega_{\mu, l} \tau\right)$, where $l$ runs over a subset of $L$ nuclei and $\mu=\uparrow, \downarrow$. If $p \ll 1$, this expression is well approximated by keeping only the $L=1$ terms, which then yields

$$
\begin{aligned}
V_{2 \mathrm{p}}(\tau) & \simeq 1-\sum_{l=1}^{l=N} \frac{p k_{l}}{4}\left[2-2 \cos \left(\omega_{\uparrow, l} \tau\right)-2 \cos \left(\omega_{\downarrow, l} \tau\right)\right. \\
& \left.+\cos \left(\left(\omega_{\uparrow, l}-\omega_{\downarrow, l}\right) \tau\right)+\cos \left(\left(\omega_{\uparrow, l}+\omega_{\downarrow, l}\right) \tau\right)\right] .
\end{aligned}
$$

One limitation of the previous pulse sequence is that the modulation envelope can only be measured up to a time of order $T_{2}$ due to electron-spin decoherence, which may be too short for appreciable spectral resolution. This limitation can be overcome by the three-pulse echo sequence shown in Fig. 1b. It consists of a $\pi / 2$ pulse applied at $t=0$ followed, after a time $\tau$ chosen such that $\tau<T_{2}$, by a second $\pi / 2$ pulse. After a variable delay $T$, a third $\pi / 2$ pulse is applied, leading to the emission of a stimulated echo at time $t=T+2 \tau$. The interest of this sequence is that the first pair of $\pi / 2$ pulses generates nuclear-spin coherence that can survive up to the nuclear-spin coherence time $T_{2, \mathrm{n}}$ which is in general much longer than $T_{2}$ (and close to the electron energy spin relaxation time $T_{1}$ ). An analytical formula can be derived for the three-pulse echo amplitude in the ideal pulse approximation (Schweiger and Jeschke, 2001):

$$
\begin{aligned}
V_{3 \mathrm{p}}(T) & =\exp \left(-T / T_{2, \mathrm{n}}\right) \exp \left(-2 \tau / T_{2}\right) \\
& \left\{1-\frac{k}{4}\left[\left[1-\cos \omega_{\downarrow} \tau\right]\left[1-\cos \omega_{\uparrow}(T+\tau)\right]\right.\right. \\
& \left.\left.+\left[1-\cos \omega_{\uparrow} \tau\right]\left[1-\cos \omega_{\downarrow}(T+\tau)\right]\right]\right\} .
\end{aligned}
$$

Contrary to two-pulse ESEEM, three-pulse echo modulation as a function of $T$ only contains the $\omega_{\downarrow}, \omega_{\uparrow}$ frequency components, and not their sum or difference; that is, in the weak-coupling limit $A, B \ll \omega_{I}$, only the nuclear-spin Larmor frequency $\omega_{I}$ appears in the spectrum. Another difference is that the modulation pattern and amplitude depend on $\tau$; in particular, its amplitude is zero whenever $\omega_{\downarrow, \uparrow} \tau=2 \pi n$ with $n$ integer ("blind spots").

For weakly coupled nuclei, the modulation amplitude of three-pulse ESEEM can be enhanced by up to 1 order of magnitude by using a more complex pulse sequence known as five-pulse ESEEM (Schweiger and Jeschke, 2001; Kasumaj and Stoll, 2008) and shown in Fig. 1. The analytical formula for the five-pulse echo amplitude $V_{5 \mathrm{p}}$ is given in the Supplement.

Equation (6), with proper modification to take into account contributions of different pathways, can be applied to the three- and five-pulse ESEEM to treat coupling to multiple nuclear spins. The details are shown in Sect. S3.3 of the Supplement.

\subsection{Fictitious spin model}

The electronic spins that we consider in this work involve an unpaired electron with spin of $S_{0}=1 / 2$ either located 
around or trapped by an ionic defect, which itself can possess a non-zero nuclear spin of $I_{0}$. These two spins of the defect are strongly coupled and therefore form a multi-level system, which can nevertheless be mapped to an effective, fictitious, spin-1/2 model as explained below (Schweiger and Jeschke, 2001), to which the model of Sect. 2.3 can be applied.

The system spin Hamiltonian writes

$$
\mathbf{H}_{\text {ion }}=\beta_{\mathrm{e}} \boldsymbol{B}_{\mathbf{0}} \cdot \mathbf{g}_{\mathrm{e}} \cdot \mathbf{S}_{0}+\mathbf{S}_{0} \cdot \mathbf{A}_{0} \cdot \mathbf{I}_{0} .
$$

Here, $\beta_{\mathrm{e}}$ is the electron Bohr magneton, $\mathbf{g}_{\mathrm{e}}$ is the (possibly anisotropic) gyromagnetic tensor, and $\mathbf{A}_{0}$ the hyperfine tensor. The nuclear Zeeman interaction of the defect system, being small compared to the hyperfine interaction in the range of magnetic fields explored here, is neglected from the Hamiltonian.

This multi-level electron-spin system is coupled to other nuclear spins in the lattice, giving rise to ESEEM. Consider a nuclear spin at a lattice site $j$, defined by its location $\boldsymbol{r}_{j}$ with respect to the electron spin. The nuclear Zeeman Hamiltonian is $\mathbf{H}_{j}=\omega_{I} \mathbf{I}_{j, z}$, with $\omega_{I}=g_{\mathrm{n}} \beta_{\mathrm{n}} B_{0}, g_{\mathrm{n}}$ being the nuclear $g$ factor and $\beta_{\mathrm{n}}$ the nuclear magneton. Its hyperfine coupling to the electron-spin system is described by the Hamiltonian:

$\mathbf{H}_{j, \mathrm{hf}}=\mathbf{S}_{0} \cdot \mathbf{A}_{j} \cdot \mathbf{I}_{j}$,

with

$\mathbf{A}_{j}=\mathbf{A}_{j, \mathrm{cf}}+\mathbf{A}_{j, \mathrm{dd}}$.

This hyperfine tensor consists of a Fermi contact term, $\mathbf{A}_{j, \mathrm{cf}}=\frac{2}{3} \mu_{0} \beta_{\mathrm{e}} g_{\mathrm{n}} \beta_{\mathrm{n}} \mathbf{g}_{\mathrm{e}}\left|\psi\left(\boldsymbol{r}_{j}\right)\right|^{2}$, and a dipole-dipole term, $\mathbf{A}_{j, \mathrm{dd}}=\frac{3 \mu_{0}}{4 \pi\left|\boldsymbol{r}_{j}\right|^{5}} \beta_{\mathrm{e}} \beta_{\mathrm{n}} g_{\mathrm{n}}\left[\boldsymbol{r}_{j}^{2} \mathbf{g}_{\mathrm{e}}-3\left(\mathbf{g}_{\mathrm{e}} \cdot \boldsymbol{r}_{j}\right) \boldsymbol{r}_{j}\right], \psi\left(\boldsymbol{r}_{j}\right)$, being the electron wave function at the nuclear-spin location.

The Hamiltonian $\mathbf{H}_{\text {ion }}$ (Eq. 10) can be diagonalized, yielding $4 I_{0}+2$ energy levels. It is in general possible to isolate two levels $|\alpha\rangle$ and $|\beta\rangle$ that are coupled by an electron spin resonance (ESR)-allowed transition and are resonant or quasi-resonant with the microwave cavity, with a transition frequency $\omega_{S}$. If these two levels are sufficiently separated in energy from other levels of $\mathbf{H}_{\text {ion }}$, they define a fictitious $S=1 / 2$ system. Writing the total Hamiltonian $\mathbf{H}_{\text {ion }}+\mathbf{H}_{j}+\mathbf{H}_{j \text {, hf }}$ restricted to this two-dimensional subspace yields

$$
\begin{aligned}
\mathbf{H}_{0} & =\omega_{S} \mathbf{S}_{z}+\left(\omega_{I}+\frac{m_{S}^{\alpha}+m_{S}^{\beta}}{2} \mathbf{A}_{j, z z}\right) \mathbf{I}_{j, z} \\
& +\frac{m_{S}^{\alpha}+m_{S}^{\beta}}{2} \mathbf{A}_{j, z x} \mathbf{I}_{j, x} \\
& +\left(m_{S}^{\alpha}-m_{S}^{\beta}\right)\left(\mathbf{A}_{j, z z} \mathbf{S}_{z} \mathbf{I}_{j, z}+\mathbf{A}_{j, z x} \mathbf{S}_{z} \mathbf{I}_{j, x}\right)
\end{aligned}
$$

where $m_{S}^{\alpha, \beta}=\left\langle\alpha, \beta\left|\mathbf{S}_{0, z}\right| \alpha, \beta\right\rangle$.

Equation (13) maps the more complex system to the simple model of Sect. 2.2. Compared to Eq. (2), two differences appear. First, the hyperfine interaction parameters $A, B$ are rescaled by the effective longitudinal magnetization difference $\left(m_{S}^{\alpha}-m_{S}^{\beta}\right)$ which depends on the two levels considered. Second, when the average longitudinal magnetization of the two levels $\left(m_{S}^{\alpha}+m_{S}^{\beta}\right)$ is non-zero, the nuclear spin sees an extra Zeeman contribution which may be tilted with respect to the $z$ axis. Once taken into account, these corrections, the analysis, and formulas of Sect. 2.3 remain valid.

\section{Spin systems}

\subsection{Erbium-doped $\mathrm{CaWO}_{4}$}

The first system investigated consists of erbium $\mathrm{Er}^{3+}$ ions doped into a $\mathrm{CaWO}_{4}$ matrix, substituting $\mathrm{Ca}^{2+}$. The crystal has a tetragonal body-centered structure (see Fig. 3) with lattice constants $a=b=0.524 \mathrm{~nm}$ and $c=1.137 \mathrm{~nm}$. Rareearth ions with an odd number of electrons such as $\mathrm{Er}^{3+}$ have a ground state consisting of two levels that are degenerate in zero magnetic field and separated from other levels by an energy scale equivalent to several tens of Kelvin due to the crystalline electric field and the spin-orbit interaction. This pair of electronic levels is known as a Kramers doublet and forms an effective $S_{0}=1 / 2$ electron-spin system, with a spin Hamiltonian $\mathbf{H}_{\mathrm{Er}}$ (Abragam and Bleaney, 2012) whose form is given by Eq. (10).

Due to the S4 site symmetry in which rare-earth ions are found in $\mathrm{CaWO}_{4}$, the $g$ tensor is diagonal in the crystallographic frame with $g_{x x}=g_{y y}=8.38$ and $g_{z z}=1.247$ (Antipin et al., 1968) ( $x, y, z$ corresponding to $a, b, c)$. Of all erbium atoms, $77 \%$ are from an isotope that has nuclear spin of $I_{0}=0$ and therefore no contribution from the hyperfine term in Eq. (10). Their energy levels are shown in Fig. 3 for $B_{0}$ applied in the $(a, b)$ plane.

The remaining $23 \%$ are from the ${ }^{167} \mathrm{Er}$ isotope with $I_{0}=7 / 2$. Its hyperfine coupling tensor to the $\mathrm{Er}^{3+}$ electron spin is diagonal, with coefficients $A_{x x}=A_{y y}=873 \mathrm{MHz}$ and $A_{z z}=130 \mathrm{MHz}$. The 16 eigenfrequencies of the ${ }^{167} \mathrm{Er}$ spin Hamiltonian are also shown in Fig. 3, again for $B_{0}$ applied in the $(a, b)$ plane. In the high-magnetic-field limit of $B_{0} \gg A_{\mathrm{Er}} /\left(g_{\mathrm{Er}} \beta_{\mathrm{e}}\right)$, the eigenstates are simply described by $\left| \pm, m_{I}\right\rangle, \pm$ describing the electron-spin quantum number $m_{S}= \pm 1 / 2$ and $m_{I}$ the nuclear-spin quantum number. For $B_{0}<100 \mathrm{mT}$, as is the case in the measurements described here, this limit is only approximate, but we will nevertheless use the high-field state vectors as labels for the lowerfield eigenstates. The strongest EPR-allowed transitions are the $m_{I}$-preserving transitions. In the following, we will apply the fictitious spin model with $|\alpha, \beta\rangle=\left| \pm, m_{I}\right\rangle$.

The $\mathrm{CaWO}_{4}$ matrix also contains nuclear spins. Indeed, the ${ }^{183} \mathrm{~W}$ isotope has a spin of $I=1 / 2$ with nuclear $g$ factor $g_{\mathrm{n}}=0.235$ (corresponding to a gyromagnetic ratio of $1.8 \mathrm{MHz} \mathrm{T}^{-1}$ ) and is present in a $p=0.13$ abundance, whereas the other tungsten isotopes are nuclear-spin free. The interaction of the ${ }^{183} \mathrm{~W}$ atoms with the erbium ions gives 
(a)

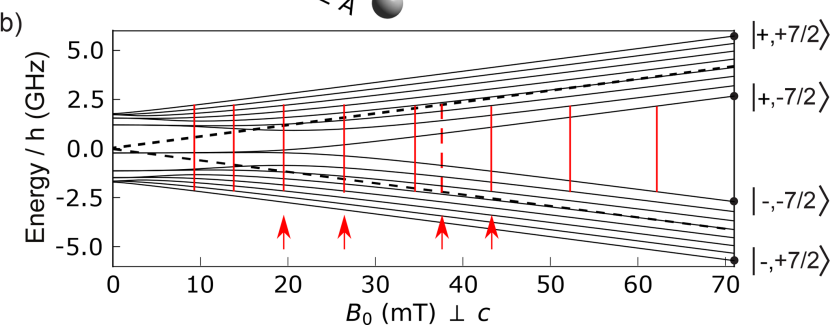

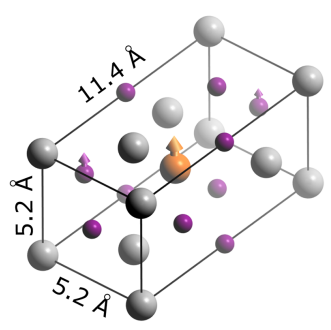

a

$\mathrm{Ca}^{2+}$

- W

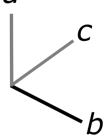

$+,+7 / 2\rangle$

Figure 3. Structure and energy diagram of erbium ions in $\mathrm{CaWO}_{4}$. (a) Crystal structure with oxygen atoms hidden for clarity. Erbium atoms are in substitution of the calcium. The crystal has a rotational symmetry around the $c$ axis. A fraction $p=0.13$ of the $W$ atoms are of the ${ }^{183} \mathrm{~W}$ isotope, with a nuclear spin of $1 / 2$. (b) Energy level diagram of the $I=0$ erbium isotopes (dashed black line) and of the ${ }^{167} \mathrm{Er}$ isotope (solid black lines) with $I=7 / 2$ for $B_{0}$ applied perpendicular to the $c$ axis. Vertical red lines indicate the value of $B_{0}$ for which an allowed EPR transition becomes resonant with the $4.372 \mathrm{GHz}$ frequency of our detection resonator (see main text, Sect. 4). Four red arrows indicate the values of $B_{0}$ at which ESEEM data were measured.

rise to the ESEEM studied below. Because the $4 \mathrm{f}$ electron wave function is mainly located on the $\mathrm{Er}^{3+}$ ion, the contact hyperfine with the nuclear spins of the lattice is expected to be negligibly small. We therefore model the hyperfine interaction with ${ }^{183} \mathrm{~W}$ by the dipole-dipole term in Eq. (12).

\subsection{Bismuth donors in silicon}

The other system considered is the bismuth donor in silicon. Bismuth, as an element of the fifth column, substitutes in the silicon lattice by making four covalent bonds with neighboring atoms, leaving one unpaired electron that can be weakly trapped by the hydrogenic potential generated by the $\mathrm{Bi}^{+}$ion, whose spin gives rise to the resonance signal (see Fig. 4a). The donor wave function $\psi(\boldsymbol{r})$ has a complex structure that extends over $\approx 1.5 \mathrm{~nm}$ in the silicon lattice (Kohn and Luttinger, 1955; Feher, 1959) (see the Supplement). As for $\mathrm{Er}: \mathrm{CaWO}_{4}$, the donor spin Hamiltonian $\mathbf{H}_{\mathrm{Bi}}$ is given by Eq. (10). However, in this case, the $g$ tensor $g_{\mathrm{e}} \mathbf{1}$ is isotropic with $g_{\mathrm{e}}=2$, and the hyperfine tensor $A_{\mathrm{Bi}} \mathbf{1}$ with the nuclear spin of $I_{0}=9 / 2$ of the bismuth atom is also isotropic, with $A_{\mathrm{Bi}} / 2 \pi=1.4754 \mathrm{GHz}$.

The eigenstates of $\mathbf{H}_{\mathrm{Bi}}$ have simple properties because of its isotropic character. Denoting $m_{S}\left(m_{I}\right)$ the eigenvalue of $\mathbf{S}_{z, 0}\left(\mathbf{I}_{z, 0}\right)$, we note that $m=m_{I}+m_{S}$ is a good quantum number since $\mathbf{H}_{\mathrm{Bi}}$ commutes with $\mathbf{S}_{z, 0}+\mathbf{I}_{z, 0}$ (Mohammady

et al., 2010), $z$ being the direction of $\boldsymbol{B}_{\mathbf{0}}$. States with equal $m$ are hybridized by $\mathbf{H}_{\mathrm{Bi}}$. States $|m=5\rangle$ and $|m=-5\rangle$, corresponding to $\left|m_{S}=+1 / 2, m_{I}=9 / 2\right\rangle$ and $\mid m_{S}=-1 / 2, m_{I}=$ $-9 / 2\rangle$, are non-degenerate and are thus also eigenstates of $\mathbf{H}_{\mathrm{Bi}}$. States with $|m| \leq 4$ belong to nine two-dimensional subspaces spanned by $\left|m_{S}=+1 / 2, m_{I}=m-1 / 2\right\rangle, \mid m_{S}=$ $\left.-1 / 2, m_{I}=m+1 / 2\right\rangle$ within which the two eigenstates of $\mathbf{H}_{\mathrm{Bi}}$ are given by $| \pm, m\rangle=a_{m}^{ \pm}\left| \pm \frac{1}{2}, m \mp \frac{1}{2}\right\rangle+b_{m}^{ \pm}\left|\mp \frac{1}{2}, m \pm \frac{1}{2}\right\rangle$, with values of $a_{m}^{ \pm}, b_{m}^{ \pm}$that can be determined analytically (Mohammady et al., 2010).

Contrary to the erbium case, the measurements of bismuth donor spins are performed in the low-field limit $\left|g_{\mathrm{e}} \beta_{\mathrm{e}} B_{0}\right| \ll$ $\left|A_{\mathrm{Bi}}\right|$, in which the eigenstates are fully hybridized. In this limit, a useful approximate expression for the eigenenergy of level $| \pm, m\rangle$ is

$E_{m}^{ \pm} \approx-\frac{A_{\mathrm{Bi}}}{2} \pm \frac{5 A_{\mathrm{Bi}}}{2} \pm \frac{m g_{\mathrm{e}} \beta_{\mathrm{e}}\left|\boldsymbol{B}_{0}\right|}{10}$

The magnetic-field dependence of the $| \pm, m\rangle$ energy levels is shown in Fig. $4 \mathrm{~b}$ for $B_{0}<1 \mathrm{mT}$. Note in particular that the separation between neighboring hyperfine levels is given by $E_{m}^{ \pm}-E_{m-1}^{ \pm} \approx \pm \frac{g_{\mathrm{e}} \beta_{\mathrm{e}} B_{0}}{10}= \pm 2 \pi \times 2.8 B_{0} \mathrm{GHz}$

Because of the hybridization, all transitions that satisfy $|\Delta m|=1$ are to some extent EPR allowed at low field; i.e., they have a non-zero matrix element of operator $S_{0, x}$. In this work, we particularly focus on the $18|\Delta m|=1$ transitions that are in the $\simeq 7 \mathrm{GHz}$ frequency range at low magnetic fields $|+, m\rangle \leftrightarrow|-, m-1\rangle$ and $|-, m\rangle \leftrightarrow|+, m-1\rangle$, as shown in Fig. 4c. The $|-, m\rangle \leftrightarrow|+, m+1\rangle$ and $\mid-, m+$ $1\rangle \leftrightarrow|+, m-1\rangle$ transitions are degenerate in frequency for $-4 \leq m<4$ as seen from Eq. (14), which results in only 10 different transition frequencies (see Figs. $4 \mathrm{~b}-\mathrm{c}$ and $8 \mathrm{a}$ ).

The most abundant isotope of silicon is ${ }^{28} \mathrm{Si}$, which is nuclear-spin free. The lattice also contains a small percentage $p$ of ${ }^{29} \mathrm{Si}$ atoms that have a nuclear spin of $I=1 / 2$ and give rise to the ESEEM. The $g$ factor of ${ }^{29} \mathrm{Si}$ is $g_{\mathrm{n}}=-1.11$, yielding a gyromagnetic ratio of $8.46 \mathrm{MHz} \mathrm{T}^{-1}$.

The donor $-{ }^{29} \mathrm{Si}$ hyperfine interaction is given by Eq. (12). Due to the spatial extent of the electron wave function, the Fermi contact term is not negligible and needs to be taken into account together with the dipole-dipole coupling (Hale and Mieher, 1969); more details can be found in the Supplement.

The restriction of the total system Hamiltonian to each of the 18 ESR-allowed transitions of the bismuth donor manifold can be mapped onto the fictitious spin-1/2 model of Sect. 2.4. Note however that the hyperfine term $\left|A_{j}\right|$ can take values up to $\sim 1 \mathrm{MHz}$ for proximal nuclear spins, which is comparable to or larger than the frequency difference between hyperfine states of the bismuth donor manifold at low field as explained above. The validity of the fictitious spin$1 / 2$ model in this context will be discussed in Sect. 5 . 

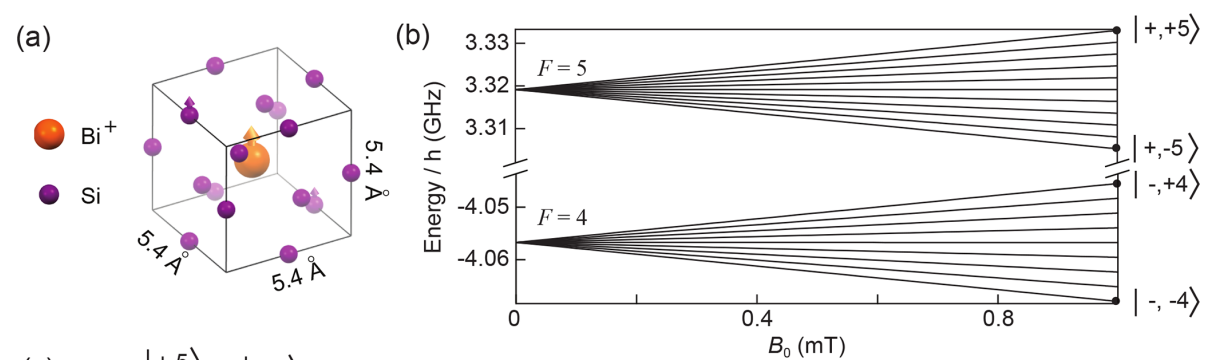

(c)

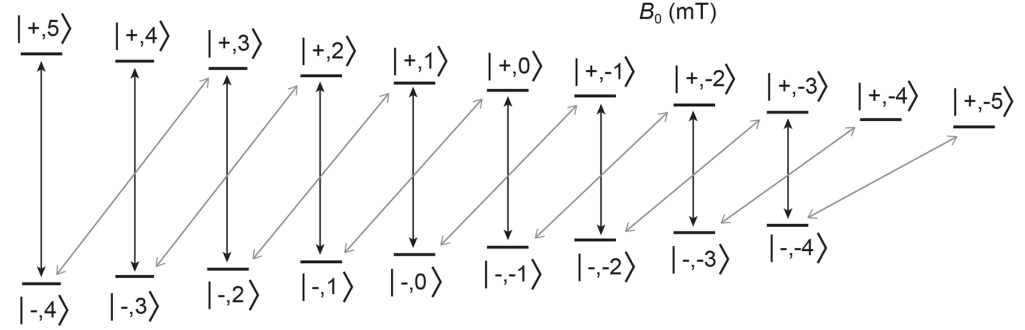

Figure 4. Structure and energy diagram of bismuth donors in silicon. (a) Silicon crystal structure, showing a substitutional bismuth atom coupled to nearby ${ }^{29} \mathrm{Si}$ nuclear spins. The donor electron is trapped around the $\mathrm{Bi}^{+}$ion and its wave function covers many lattice sites. (b) Energy levels of the bismuth donor for $B_{0}<1 \mathrm{mT}$. (c) Schematic representation of the allowed transitions (black and grey arrows) between the bismuth donor energy levels in the low field limit.

\section{Experimental setup and samples}

The EPR spectrometer has been described in detail in Bienfait et al. (2015) and Probst et al. (2017) and is shown schematically in Fig. 5a. It is built around a superconducting micro-resonator of frequency $\omega_{\mathrm{r}}$ consisting of a planar interdigitated capacitor shunted by an inductor, directly patterned on the crystal. We detect the spins that are located in the immediate vicinity of the resonator inductance. Note that the microwave $B_{1}$ field generated by the inductance is spatially inhomogeneous. If the spin location is broadly distributed, this can make the application of control pulses with a well-defined Rabi angle problematic (Ranjan et al., 2020a). As explained below, the resonator is more strongly coupled to the measurement line than in Bienfait et al. (2015) to increase the measurement bandwidth as requested for ESEEM spectroscopy.

The sample is mounted in a copper sample holder thermally anchored at the mixing chamber of a dilution refrigerator. A DC magnetic field $B_{0}$ is applied parallel to the sample surface and along the resonator inductance. The resonator is coupled capacitively to an antenna, which is itself connected to a microwave measurement setup in reflection. To minimize heat load, the coaxial cables between $4 \mathrm{~K}$ and $10 \mathrm{mK}$ are in superconducting niobium-titanium (NbTi). To suppress thermal noise, the input line is heavily attenuated at low temperatures. Microwave pulses for driving the spins are sent to the resonator input, and their reflection or transmission, together with the echo signal emitted by the spins, is fed into a superconducting JPA, either of the flux-pumped type (Zhou et al., 2014) or of the Josephson traveling-wave parametric amplifier (JTWPA) type (Macklin et al., 2015). Further microwave amplification takes place at $4 \mathrm{~K}$ with a high- electron-mobility transistor (HEMT) from Low Noise Factory, and then at room temperature, before homodyne demodulation which yields the two signal quadratures $[I(t), Q(t)]$. The echo-containing quadrature signal is integrated to yield the echo amplitude $A_{\mathrm{e}}$. Such a setup was shown to reach sensitivities of the order of $10^{2}-10^{3} \mathrm{spin} / \sqrt{\mathrm{Hz}}$ (Bienfait et al., 2015; Eichler et al., 2017; Probst et al., 2017).

Because of the small resonator mode volume and high quality factor, little microwave power is needed to drive the spins. The exact amount depends on the resonator geometry, as conveniently expressed by the power-to-field conversion factor $\alpha=B_{1} / \sqrt{P_{\text {in }}}$. In the experiments reported here, the maximum microwave power used to drive the spins is on the order of $10 \mathrm{nW}$. At this power, the superconducting pre-amplifiers saturate; however, they recover rapidly enough (within a few microseconds) to amplify the much weaker subsequent spin echoes. Flux-pumped JPAs are moreover switched off during the control pulses by pulsing the pump tone, whereas the JTWPA was kept on all the time. All microwave powers reaching the $4 \mathrm{~K}$ HEMT are low enough that neither saturation nor damage are to be expected at this stage.

The erbium-doped sample (from Scientific Materials) was prepared by mixing erbium oxide with calcium and tungsten oxides before crystal growth, yielding a uniform Er concentration of $6 \times 10^{17} \mathrm{~cm}^{-3}(50 \mathrm{ppm})$ throughout the sample. For resonator fabrication, the bulk crystal was cut and polished to a thin rectangular sample with dimensions $0.4, \mathrm{~mm} \times$ $3 \mathrm{~mm} \times 6$, mm parallel to $a \times b \times c$ axes. The resonator was patterned out of a $100 \mathrm{~nm}$ thick (sputtered) niobium $(\mathrm{Nb})$ layer, using a design similar to that shown in Bienfait et al. (2015). More specifically, 15 interdigitated fingers on either side of a $720 \mu \mathrm{m} \times 5 \mu \mathrm{m}$ inductive wire form an LC res- 
(a) $\omega_{r}$

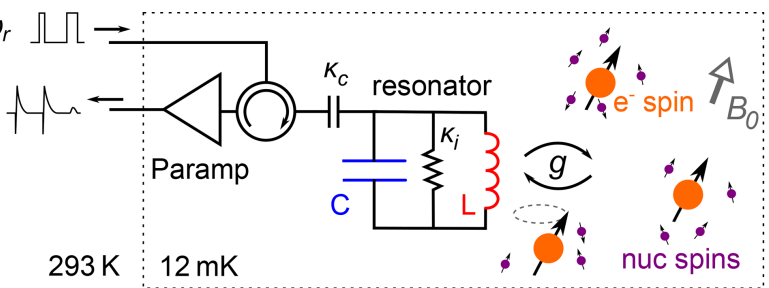

(b)

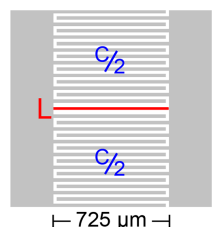

(c)

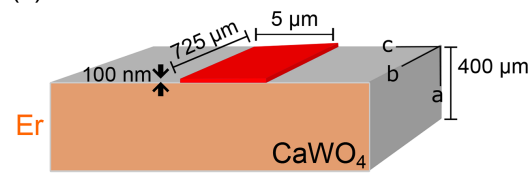

(d)

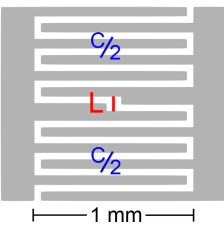

(e)

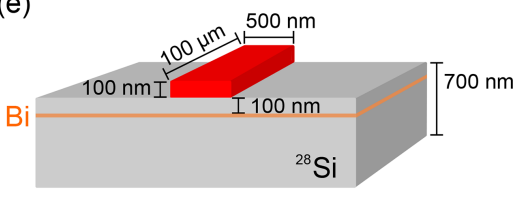

Figure 5. Experimental setup and samples. (a) Schematic of the low-temperature EPR spectrometer. The LC resonator is inductively coupled to electron spins, which are coupled to a nuclear-spin bath that causes the ESEEM. The spins are probed by sequences of microwave pulses at the resonator frequency $\omega_{\mathrm{r}}=1 / \sqrt{\mathrm{LC}}$. Reflected pulses as well as the echo signal are routed to a parametric amplifier, further amplified at $4 \mathrm{~K}$, and finally demodulated and digitized at room temperature. $(\mathbf{b}, \mathbf{c})$ Design of the LC resonator used for the detection of erbium ion spins, with a $725 \mu \mathrm{m}$ long, $5 \mu \mathrm{m}$ wide inductor. It is patterned out of a $100 \mathrm{~nm}$ thick niobium film deposited on top of a $\mathrm{CaWO}_{4}$ substrate bulk doped with $\mathrm{Er}^{3+}$ ions. (d, e) Design of the LC resonator used for the detection of bismuth donor spins, with a $100 \mu \mathrm{m}$ long, $0.5 \mu \mathrm{m}$ wide inductor. It is patterned out of a $100 \mathrm{~nm}$ thick aluminum film deposited on top of a silicon substrate isotopically enriched in ${ }^{28} \mathrm{Si}$, in which bismuth ions were implanted at a $50-100 \mathrm{~nm}$ depth.

onator, corresponding to a detection volume of $V_{\mathrm{Er}} \sim 20 \mathrm{pL}$. In the absence of magnetic field, the resonance frequency is $\omega_{\mathrm{r}} / 2 \pi=4.323 \mathrm{GHz}$. Its total quality factor of $8 \times 10^{3}$ is set both by the internal losses, characterized by the energy loss rate $\kappa_{\mathrm{i}}=5 \times 10^{5} \mathrm{~s}^{-1}$, and by its coupling to the measurement line $\kappa_{\mathrm{C}}=3 \times 10^{6} \mathrm{~s}^{-1}$. For this geometry, the power-to-field factor is $\alpha=1.7 \mathrm{~T} \mathrm{~W}^{-1 / 2}$.

The bismuth donors have been implanted at $\approx 100 \mathrm{~nm}$ depth with a peak concentration of $8 \times 10^{16} \mathrm{~cm}^{-3}$ in a silicon sample. They lie in a $700 \mathrm{~nm}$ thick silicon epilayer enriched in the nuclear-spin-free ${ }^{28} \mathrm{Si}$ isotope (nominal concentration of $99.95 \%$ ), grown on top of a natural-abundance silicon sample. The resonator is patterned out of a $50 \mathrm{~nm}$ thick aluminum film. It has the same geometry as reported in Probst et al. (2017), with a $100 \mu \mathrm{m}$ long, $500 \mathrm{~nm}$ wide inductor, and a detection volume of $0.2 \mathrm{pL}$. Its frequency $\omega_{\mathrm{r}} / 2 \pi=$ $7.370 \mathrm{GHz}$ is only slightly below the zero-field splitting

of unperturbed $\mathrm{Bi}: \mathrm{Si}$ donors at $5 A_{\mathrm{Bi}} /(2 \pi)=7.37585 \mathrm{GHz}$ (Wolfowicz et al., 2013). The resonator internal loss is given by $\kappa_{\mathrm{i}}=3 \times 10^{5} \mathrm{~s}^{-1}$. The coupling to the measurement line can be tuned at will by modifying the length of a microwave antenna that capacitively couples the measurement waveguide to the on-chip resonator via the copper sample holder (Bienfait et al., 2015; Probst et al., 2017). For the experiments reported below, we used two settings: one for which the resonator was overcoupled $\left(\kappa_{\mathrm{C} 1}=10^{7} \mathrm{~s}^{-1}\right)$, corresponding to a loaded quality factor of $Q_{1}=4 \times 10^{3}$, and one for which the coupling was closer to critical $\left(\kappa_{\mathrm{C} 2}=10^{6} \mathrm{~s}^{-1}\right)$, corresponding to a loaded quality factor of $Q_{2}=3.4 \times 10^{4}$. In the low- $Q$ case, square microwave pulses were used, of duration $\simeq 100$ ns similar to the cavity field damping time. In the high- $Q$ case, shaped pulses were used (Probst et al., 2019) so that the intra-cavity field was a square pulse of $1 \mu \mathrm{s}$ without any ringing. In some experiments, we additionally used a train of $\pi$ pulses (Carr-Purcell-Meiboom-Gill sequence; CPMG), which generated extra echoes for significant gain in signal-to-noise ratio. More details on the pulse sequences used, the phase cycling scheme, and the repetition time, will be given in the following sections, together with experimental results. For this geometry, the power-to-field factor is $\alpha=9 \mathrm{~T} \mathrm{~W}^{-1 / 2}$ for the low- $Q$ case, and $\alpha=21 \mathrm{~T} \mathrm{~W}^{-1 / 2}$ for the high- $Q$ case.

\section{Results}

\subsection{Erbium-doped $\mathrm{CaWO}_{4}$}

\subsubsection{Spectroscopy}

Figure 6 shows a spectrum comprising a series of microwave transmission measurements recorded on a vector network analyzer, measured at $100 \mathrm{mK}$, as a function of the magnetic field $B_{0}$ applied along the $b$ crystal axis (Probst et al., 2020). Note that compared to Fig. 5a, the resonator is coupled to the measurement line in a hanger geometry (Day et al., 2003), so that its resonance appears as a dip in the amplitude transmission coefficient $\left|S_{21}\right|$ (see Fig. 6). The nine red lines indicate the values of $B_{0}$ at which the calculated $\mathrm{Er}^{3+}$ ion transitions are equal to $\omega_{\mathrm{r}}$ (see Fig. 3b). Avoided level crossings are observed, which indicate a strong coupling of the resonator to the erbium transitions. Several additional anti-crossings and discontinuities are visible above $40 \mathrm{mT}$. These are attributed to ytterbium impurities ${ }^{171} \mathrm{Yb}$ and ${ }^{173} \mathrm{Yb}$ and magnetic flux vortices penetrating the resonator.

Noticeable in the spectrum at $37 \mathrm{mT}$ is the large anticrossing attributed to the highly concentrated $I=0$ erbium isotopes. Here, the high-cooperativity regime $(C>30)$ is reached between the electronic spins and the resonator (Kubo et al., 2010; Probst et al., 2013). Typical linewidth of $\Gamma / 2 \pi \sim$ $20 \mathrm{MHz}$ is observed. The coupling strength is also observed to be different for the eight ${ }^{167} \mathrm{Er}$ transitions, which are labeled according to their corresponding nuclear-spin projec- 


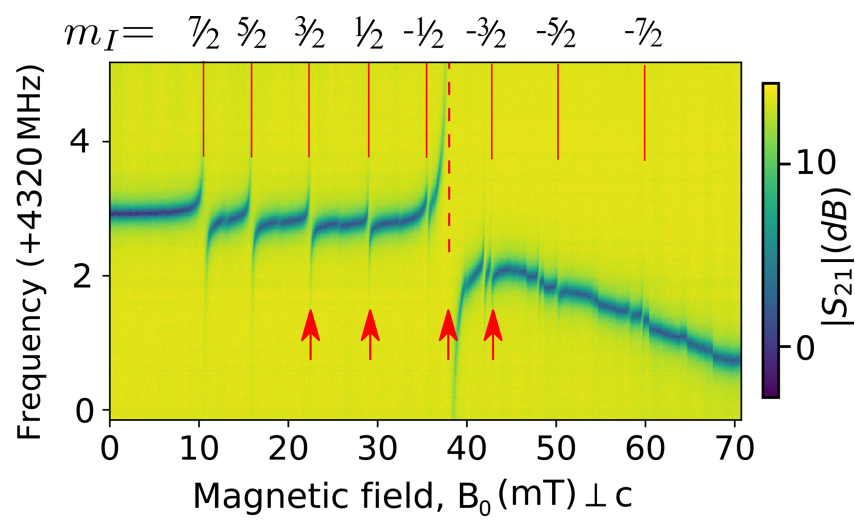

Figure 6. Spectroscopy of $\mathrm{Er}^{3+}: \mathrm{CaWO}_{4}$. Transmission coefficient $\left|S_{21}\right|(\omega)$ at $100 \mathrm{mK}$ as a function of the magnetic field $B_{0}$ applied along to the $a$ crystalline axis, around $4.323 \mathrm{GHz}$. Vertical red lines indicate the expected erbium transitions either for the $I=0$ isotopes (dashed) or the $I=7 / 2$ isotope (solid). Red arrows indicate the field at which the ESEEM data are measured.

tions $m_{I}$. This is explained by the partial polarization of the ground-state hyperfine levels of ${ }^{167} \mathrm{Er}^{3+}$ at millikelvin temperatures (see Fig. 3b).

\subsubsection{Two-pulse ESEEM}

Four values of $B_{0}$ were selected for investigating ESEEM, indicated by the arrows in Fig. 6; the first, second, and fourth corresponding to electronic-spin transitions of ${ }^{167} \mathrm{Er}$, and the third one to the $I=0$ isotopes. The two-pulse echo sequence of Fig. 1a was implemented with square pulses of $1 \mu$ s duration applied at the resonator input, with double amplitude for the second pulse. Note that due to the $B_{1}$ spatial inhomogeneity combined with the homogeneous spin distribution throughout the crystal, the spread of Rabi frequency is too large to observe a well-defined nutation signal. The Rabi angle is therefore not well defined, and the echo is the average of different rotation angles.

The control pulses driving the spins are filtered by the resonator bandwidth $\kappa / 2 \pi \simeq 600 \mathrm{kHz}$, corresponding to a field decay time $2 \kappa^{-1}=3.3 \mu \mathrm{s}$. The repetition time between echo sequences was $1 \mathrm{~s}$, close to the spin relaxation time $T_{1} \sim 1-$ $2 \mathrm{~s}$ measured by saturation recovery on the transitions studied. The echo signal was averaged 10 times with phase cycling of the $\pi$ pulse to improve signal-to-noise ratio and to remove signal offsets.

Figure 7 shows the two-pulse echo integrated amplitude $A_{\mathrm{e}}$ as a function of $\tau$ for each of the four Er transitions investigated (Probst et al., 2020). A clear envelope modulation signal is observed, together with an overall damping. Here, we are interested only in the modulation pattern; a detailed study of the coherence time $T_{2}$ will be provided elsewhere. Qualitatively, we observe that the modulation frequency increases with $B_{0}$ and the modulation amplitude overall decreases with $B_{0}$, as expected from the discussion in Sect. 2. (a)

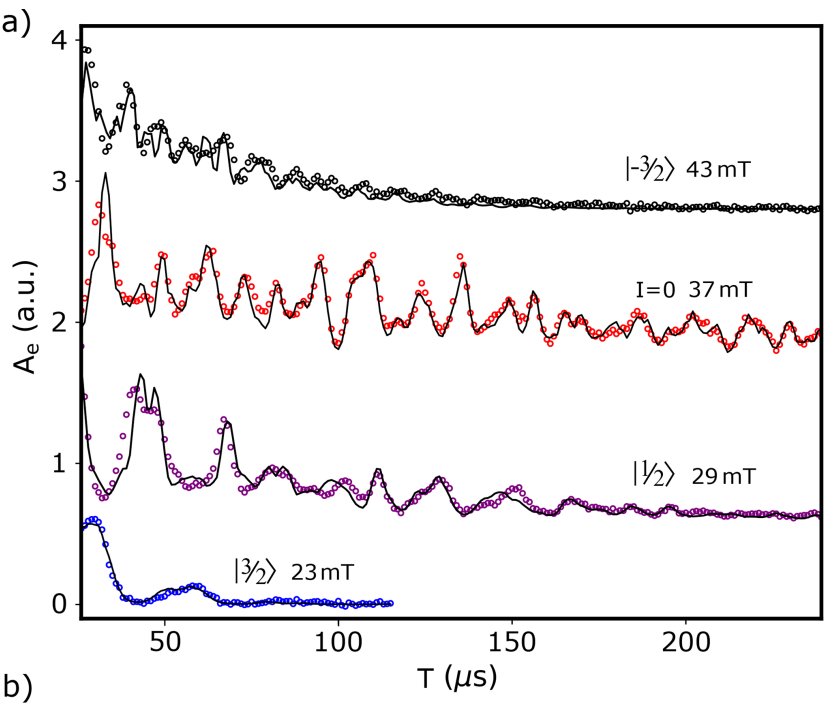

(b)

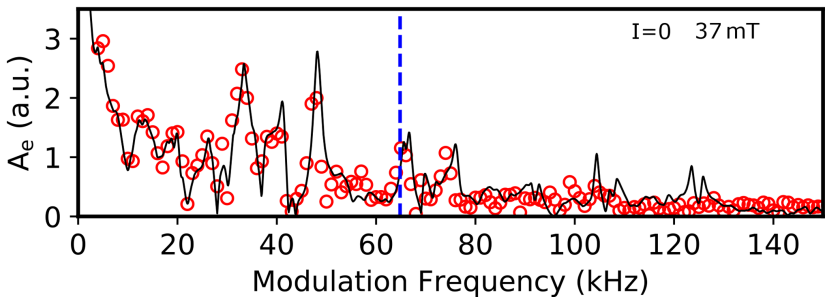

Figure 7. Two-pulse ESEEM on $\mathrm{Er}: \mathrm{CaWO}_{4}$. (a) Integrated echo area $A_{\mathrm{e}}$ as a function of the inter-pulse delay $\tau$ for four values of $B_{0}$ corresponding to different transitions. Open circles are measurements, and solid lines are the results of the ESEEM calculations as explained in Sect. S5.1. (b) Measured (open red circles) and computed (solid line) fast Fourier transform of the $I=0$ data. The dashed blue line shows the Larmor frequency of ${ }^{183} \mathrm{~W}$ nuclei in free space.

A Fourier transform of the $I=0$ data (see Fig. 7b) shows the ESEEM spectrum. Well-resolved peaks are observed in the $5-100 \mathrm{kHz}$ range, distributed around the ${ }^{183} \mathrm{~W}$ bare Larmor frequency $\omega_{\mathrm{W}}$.

A very rough estimate of the number of erbium ions contributing to the signal is $[\mathrm{Er}] V_{\mathrm{Er}} \kappa / \Gamma$, which is $2.5 \times 10^{8}$ for the $I=0$ data, and $10^{7}$ for each ${ }^{167} \mathrm{Er}$ transition.

\subsubsection{Comparison with the model}

We compute the echo envelope $V_{2 \mathrm{p}}^{\prime}(\tau)$ described in Sect. 2.3, with the nearest 1000 coupled tungsten nuclei $(N=1000)$ and a natural ${ }^{183} \mathrm{~W}$ abundance of $14.4 \%(p=0.144)$. The hyperfine interaction is taken to be purely dipolar, as already explained (Guillot-Noël et al., 2007; Car et al., 2018). The fitting proceeds by assigning an initial "guess" to six free parameters, then minimizing using the limited-memory Broyden-Fletcher-Goldfarb-Shanno algorithm for boundconstrained optimization (L-BFGS-B) (Byrd et al., 1995). Three of these parameters $\left(\left|B_{0}\right|, \phi, \theta\right)$ describe the applied 
magnetic field:

$\boldsymbol{B}_{0}=\left|\boldsymbol{B}_{0}\right|[\sin \theta \cos \phi \boldsymbol{x}+\sin \theta \sin \phi \boldsymbol{y}+\cos \theta \boldsymbol{z}]$.

Here, $\theta$ is the angle of the field relative to the crystal $c$ axis $(z)$ and $\phi$ is the angle relative to the $a$ axis $(\boldsymbol{x})$ in the $a-b$ plane $\left(\boldsymbol{x}-\boldsymbol{y}\right.$ plane). The other three parameters $\left(C, T_{2}, n\right)$ account for the echo envelope decay:

$1 A_{\mathrm{e}}(\tau)=V_{2 \mathrm{p}}(\tau) \cdot C \exp \left(-\frac{2 \tau}{T_{2}}\right)^{n}$,

where $C$ represents the signal magnitude, $T_{2}$ represents the coherence time, and $n \in[1,2]$ accounts for non-exponential decay. To determine the global minimum of the fit, the minimization is repeated 200 times with randomly seeded initial values for the six parameters, bounded within the known uncertainty of the applied magnetic field $B_{0}$, signal strength $C$, and coherence time $T_{2}$. This approach reveals single local minima for each fitted parameter within the bounded range, with the variance of the 200 outcomes determining the uncertainty for each parameter. In particular, it yields precise values for the angles $\theta=91.47 \pm 0.01^{\circ}$ and $\phi=90.50 \pm 0.01^{\circ}$. The result of this fitting is presented in Fig. 7a, overlaid on the data for the $I=0$ transition at $37 \mathrm{mT}$. Only the decay parameters $\left(C, T_{2}, n\right)$ and magnetic-field magnitude $\left|B_{0}\right|$ are left free when fitting the other three transitions in Fig. 7a. This was done for consistency between datasets, and because the $I=0$ data yield the most accurate values for $\phi$ and $\theta$ due to the low decoherence rate. The fits yield coherence times $T_{2}$ varying between 40 and $400 \mu$ s, depending on the transition considered. Good agreement was also reached between the fitted and expected (pre-calibrated) field magnitudes.

Note that good fits to the data are also achieved by including only the nearest 100 tungsten nuclei, although noticeable deviations between the data and fit are observed with any amount less. The dimensionless "anisotropic hyperfine interaction parameter" $\rho$ described in the seminal publication on ESEEM (Rowan et al., 1965) is not required here. This parameter was introduced with the earliest attempts of ESEEM fitting, likely to compensate for the low number of simulated nuclear spins (typically 10 nearest nuclei or less) and was interpreted as an account for a potential distortion of the local environment caused by dopant insertion. Finally, a consideration of the spectral components presented in Fig. $7 \mathrm{~b}$ helps to more clearly identify the difference between the fit and the data. In particular, the high-frequency components of the fitted model are not present experimentally due to the filtering effect of the superconducting resonance $(260 \mathrm{kHz}$ half width at half maximum; HWHM). This high- $Q$ resonator greatly reduces the bandwidth of the radio frequency (RF) field absorbed by the coupled $\mathrm{Er}-{ }^{183} \mathrm{~W}$ system and further limits the bandwidth of the detected echo signal.

\subsection{Bismuth donor sample}

\subsubsection{Spectroscopy}

Given the resonator frequency $\omega_{\mathrm{r}}$, four bismuth donor resonances should be observed when varying $B_{0}$ between 0 and $1 \mathrm{mT}$, as seen in Fig. 8a. Figure 8b shows an echodetected field sweep, measured at $12 \mathrm{mK}$ : the integrated area $A_{\mathrm{e}}$ of echoes obtained with a sequence shown in Fig. 1a with $\tau=50 \mu$ s pulse separation is plotted as a function of $B_{0}$ (Probst et al., 2020). Instead of showing well-separated peaks as in the erbium case, echoes are observed for all fields below $1 \mathrm{mT}$, with a maximum close to $0.1 \mathrm{mT}$, and extends in particular down to $B_{0}=0 \mathrm{mT}$. This is the sign that each of the expected peaks is broadened and overlaps with neighboring transitions. Close to zero field, the echo amplitude goes down by a factor of 2 on a scale of $\sim 0.1 \mathrm{mT}$, before showing a sharp increase at exactly zero field. These zero-field features are not currently understood, but they are reproducible as confirmed by the measurements at $B_{0}<0$, which are approximately symmetric to the $B_{0}>0$ data as they should be.

Line broadening was reported previously for bismuth donors in silicon in related experiments (Bienfait et al., 2015; Probst et al., 2017) and was attributed to the mechanical strain exerted by the aluminum resonator onto the silicon substrate due to differential thermal contractions between the metal and the substrate. At low strain, $A_{\mathrm{Bi}}$ depends linearly on the hydrostatic component of the strain tensor $\epsilon_{\mathrm{hs}}=\left(\epsilon_{x x}+\epsilon_{y y}+\epsilon_{z z}\right) / 3$ with a coefficient $\mathrm{d} A_{\mathrm{Bi}} / \mathrm{d} \epsilon_{\mathrm{hs}} /(2 \pi)=$ $28 \mathrm{GHz}$ (Mansir et al., 2018). Quantitative understanding of the line shape was achieved in a given sample geometry based on this mechanism (Pla et al., 2018), using a finiteelement modeling to estimate the strain profile induced upon sample cooldown. A similar modeling was performed for the Bi sample reported here (see Fig. 8d). Based on the typical strain distribution $\left|\epsilon_{\text {hyd }}\right| \sim 3 \times 10^{-4}$ and on the hyperfine to strain coefficient $\mathrm{d} A_{\mathrm{Bi}} / \mathrm{d} \epsilon_{\mathrm{hs}} /(2 \pi)=28 \mathrm{GHz}$, we expect the zero-field splitting $5 A_{\mathrm{Bi}} /(2 \pi)$ to have a spread of $\sim 50 \mathrm{MHz}$, which would indeed result in complete peak overlap in the $B_{0}<1 \mathrm{mT}$ region, as observed in Fig. $8 \mathrm{~b}$.

This broadening has two consequences worth highlighting. First, the bismuth donor echo signals can be measured down to $B_{0}=0 \mathrm{mT}$, which otherwise is generally impossible in X-band spectroscopy. Here, this is enabled by the large hyperfine coupling of the $\mathrm{Bi}$ : $\mathrm{Si}$ donor, combined with straininduced broadening. This makes it possible to detect ESEEM caused by very weakly coupled nuclear spins, which requires low magnetic fields as explained in Sect. 2. Second, at a given magnetic field, the spin-echo signal contains contributions from several overlapping EPR transitions. This last point is best understood from Fig. 8c, which shows how several classes of bismuth donors, each with different hyperfine coupling $A_{\mathrm{Bi}}$, may have transitions resonant with $\omega_{\mathrm{r}}$. We will assume in the following that the inhomogeneous distribution of $A_{\mathrm{Bi}}$ is so broad that each of the $10 A_{\mathrm{Bi}}$ values for which 

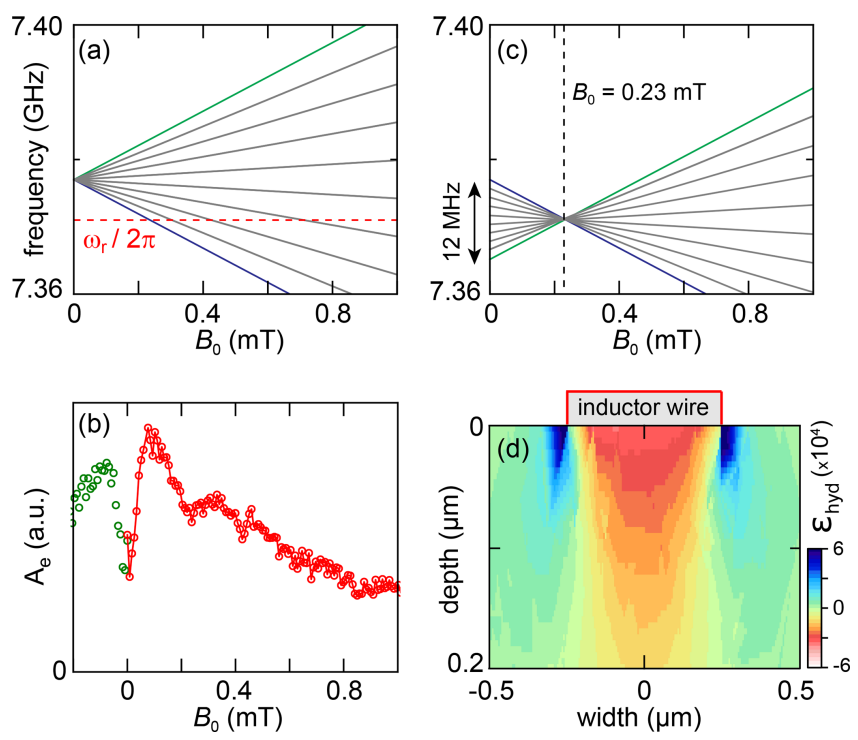

Figure 8. (a) EPR-allowed transitions of a bismuth donor in silicon for $0<B_{0}<1 \mathrm{mT}$. The dashed red line denotes the resonator frequency $\omega_{\mathrm{r}}$. The spectrum is for an unstrained donor for which the frequency at $B_{0}=0$ is $5 A_{\mathrm{Bi}} /(2 \pi)$. (b) Echo-detected field sweep. The echo integral $A_{\mathrm{e}}$ is plotted versus $B_{0}$. (c) Frequency of all 18 bismuth donor transitions that may contribute to the echo signal at a given field (here, $B_{0}=0.23 \mathrm{mT}$ ). This is made possible by the strain-induced spread in $A_{\mathrm{Bi}}$ between different donors. (d) Hydrostatic component of strain in silicon simulated using COMSOL.

one bismuth donor transition is resonant with $\omega_{\mathrm{r}}$ at fixed $B_{0}$ is equally probable, which is likely to be valid for $B_{0}<1 \mathrm{mT}$.

\subsubsection{Two-pulse ESEEM}

Two-pulse echoes are measured with the pulse sequence shown in Fig. 1, which consists of a square $\pi / 2 \mathrm{X}$ pulse of duration $50 \mathrm{~ns}$ followed by a square $\pi_{\mathrm{Y}}$ pulse of duration $100 \mathrm{~ns}$ after a delay $\tau$. Note that due to the donor spatial location in a shallow layer below the surface and to the strain shifting of their Larmor frequency (Pla et al., 2018), the Rabi frequency is more homogeneous than in the erbium-doped sample, and Rabi rotations with a well-defined angle can be applied (Pla et al., 2018; Probst et al., 2017). To increase the signal-to-noise ratio, a CPMG sequence of $198 \pi$ pulses separated by $10 \mu$ s are used following the echo sequence (Probst et al., 2017). The curves are repeated 20 times, with a delay of $2 \mathrm{~s}$ in between to enable spin relaxation of the donors. All the resulting echoes are then averaged. Phase cycling is performed by alternating sequences with opposite phases for the $\pi / 2$ pulses and subtracting the resulting echoes. The data are obtained in the low- $Q$ configuration (see Sect. 4).

Figure 9 shows the integral of the averaged echoes $A_{\mathrm{e}}(\tau)$ as a function of $\tau$ for various values of $B_{0}$ (Probst et al., 2020). At a non-zero field, $A_{\mathrm{e}}(\tau)$ shows $B_{0}$-dependent oscillations on top of an exponential decay with time constant
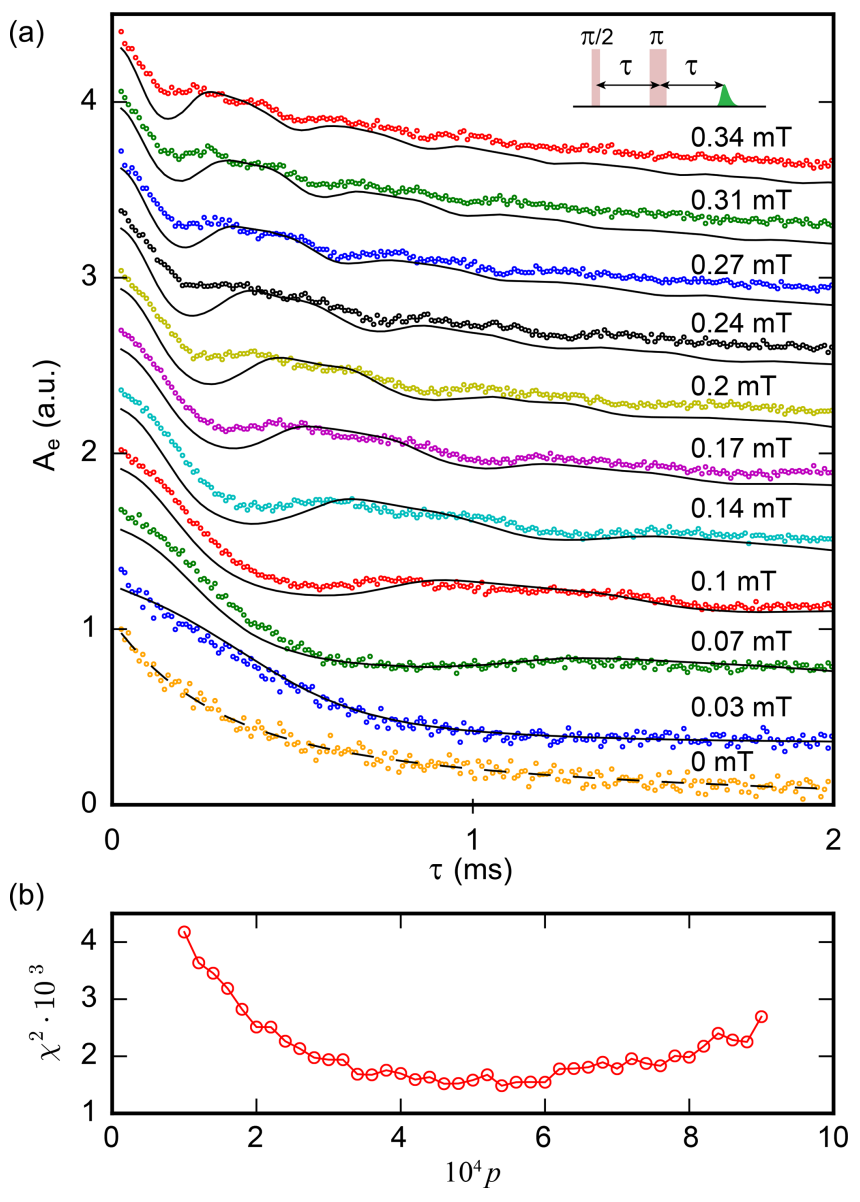

Figure 9. Two-pulse ESEEM of Bi : Si donors. (a) Echo integral $A_{\mathrm{e}}$ versus inter-pulse delay $\tau$ for a two-pulse echo sequence for varying magnetic field $B_{0}$. Dots indicate experimental data; lines indicate results of the model (see text), assuming a concentration in ${ }^{29} \mathrm{Si}$ of $p=4.4 \times 10^{-4}$. The curves are vertically shifted for clarity. (b) Fit residue $\chi^{2}$ for different ${ }^{29} \mathrm{Si}$ relative abundance $p$. The best fit is obtained for $p=4.4 \pm 1 \times 10^{-4}$, in agreement with the specified value.

$T_{2}=2.6 \mathrm{~ms}$. Similar decay times were measured on the same chip with another resonator (Probst et al., 2017), and are attributed to a combination of donor-donor dipolar interactions and magnetic noise from defects at the sample surface.

In the subsequent discussion, we concentrate on the ESEEM pattern. To analyze the data, each curve was divided by a constant exponential decay with $2.6 \mathrm{~ms}$ time constant, mirrored at $t=0$, and Fourier transformed (see Fig. 10). Only two peaks are observed. Their frequencies vary linearly with $B_{0}$, and are found to be approximately 8 and $16 \mathrm{kHz} \mathrm{mT}^{-1}$. This is in good agreement with the gyromagnetic ratio of ${ }^{29} \mathrm{Si}\left(8.46 \mathrm{kHz} \mathrm{mT}^{-1}\right)$; the presence of the second peak at twice this value is expected as explained in Sect. 2 for the two-pulse ESEEM in the weak-coupling limit. The oscillation amplitude goes down with $B_{0}$, again as expected from the model put forward in Sect. 2. 

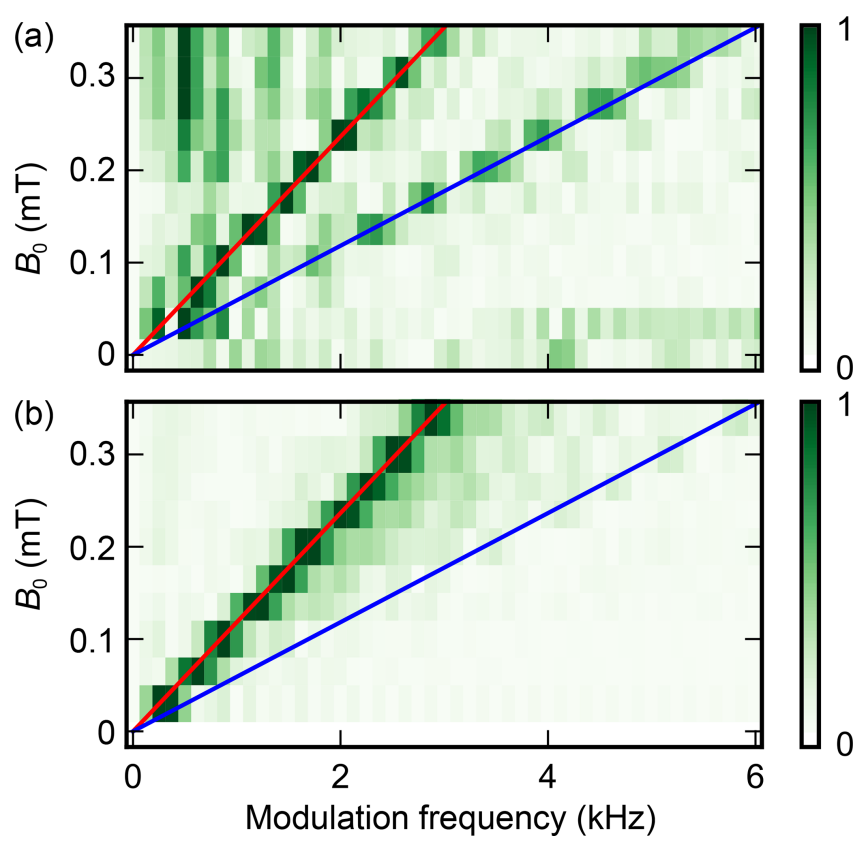

Figure 10. Amplitude of the Fourier transform of the experimental (a) and theoretical (b) two-pulse Bi : Si donor ESEEM data.

A rough estimate of the number of donors contributing to the measurements shown in Fig. 9 can be obtained by comparison with (Probst et al., 2017). Given the nearly identical resonator geometry, and assuming identical strain broadening in both samples, the ratio of the number of donors involved in both measurements is simply given by the ratio of resonator bandwidths. For the low- $Q$ configuration, such as the two-pulse echo of Fig. 9, this corresponds to $\simeq 5 \times 10^{3}$ dopants; in the high- $Q$ configuration (see the three- and fivepulse data in the next paragraph), this number is reduced to $\simeq 5 \times 10^{2}$ dopants.

\subsubsection{Three- and five-pulse ESEEM}

The spectral resolution provided by the measurement protocol is limited because of the finite electron coherence time $T_{2}$. As discussed in Sect. 2.3, this can be overcome by threeor five-pulse ESEEM.

We measure three- and five-pulse ESEEM with the pulse sequence shown in Fig. 11. The high- $Q$ configuration is chosen for which $T_{1}=120 \mathrm{~ms}$ is measured (see the Supplement); shaped pulses generate an intra-cavity field in the form of a rectangular pulse of $1 \mu$ s duration with sharp rise and fall (Probst et al., 2019) despite the high resonator quality factor. The data are acquired at $B_{0}=0.1 \mathrm{mT}$, so that $\omega_{I} / 2 \pi \simeq 850 \mathrm{~Hz}$. The first blind spot for three-pulse ESEEM is thus at $2 \pi / \omega_{I}=1.2 \mathrm{~ms}$; we chose $\tau=290 \mu \mathrm{s}$ for the three-pulse echo, and $\tau_{1}=\tau_{2}=290 \mu$ s for the five-pulse sequence. A sequence of $19 \mathrm{CPMG} \pi$ pulses, separated by $50 \mu \mathrm{s}$, was used to enhance the signal-to-noise ratio. The se- quences were repeated after a fixed waiting time of $100 \mathrm{~ms}$ between the last $\pi$ pulse of one sequence and the first $\pi / 2$ pulse of the following, to enable spin relaxation. Phase cycling is used to suppress unwanted echoes (see the Supplement for the schemes; Schweiger and Jeschke, 2001; Kasumaj and Stoll, 2008). Each point is averaged over $2.5 \times 10^{4}$ sequences, with a total acquisition time of 2 weeks for each curve (Probst et al., 2020).

The results are shown in Fig. 11, together with their fast Fourier transform (Probst et al., 2020). Both the three-pulse ESEEM (3PE) and five-pulse ESEEM (5PE) curves show oscillations that last 1 order of magnitude longer than the electron spin $T_{2}$ (up to $20 \mathrm{~ms}$ ), enabling higher spectral resolution of the ESEEM signal. The 5PE curve has a higher oscillation amplitude than the 3PE by a factor of 2-3, as expected. The decay of the oscillations occurs in $\sim 10 \mathrm{~ms}, 1$ order of magnitude faster than the stimulated echo amplitude (see the 3PE curve), suggesting that it is an intrinsic feature of the ESEEM signal, as discussed below.

The spectrum shows only one peak at the ${ }^{29} \mathrm{Si}$ frequency. This is consistent with the expression provided in Sect. 2 and the Supplement for the three- and five-pulse ESEEM, in which the terms oscillating at the sum and difference frequency are absent in contrast to the two-pulse ESEEM. The peak width is $\simeq 100 \mathrm{~Hz}$, which indicates that the nuclei contributing to the ESEEM signal have hyperfine coupling strengths $A, B$ of at most $100 \mathrm{~Hz}$. Neglecting the contact interaction term, this corresponds to ${ }^{29} \mathrm{Si}$ nuclei that are located at least $\sim 5 \mathrm{~nm}$ away from the donor spin.

The measured ESEEM spectrum of the bismuth donor sample qualitatively differs from the erbium sample, since it only contains a peak at the unperturbed silicon nuclei Larmor frequency (and at twice this frequency for the two-pulse ESEEM), instead of the many peaks observed in Fig. 7 indicating nuclear-spin contribution with vastly different hyperfine strengths. This can be qualitatively understood by examining Eq. (8). Defining $N_{l}$ as the number of lattice sites with approximately the same hyperfine parameters $A_{l}, B_{l}$ and modulation frequency $\omega_{\downarrow / \uparrow, l}$, the component at $\omega_{\downarrow / \uparrow, l}$ is visible in the spectrum if $N_{l} k_{l} p \sim 1$, which can only be achieved if $N_{l} p \sim 1$. In the case of erbium, $p=0.144$ so that even the sites closest to the ion (for which $N_{l}$ is of order unity) may satisfy this condition for well-chosen $B_{0}$. In the bismuth donor sample where $p=4.4 \times 10^{-4}$, this condition can only be met for $N_{l} \sim 10^{3}$, and therefore for crystal sites $l$ that are far from the donor, for which the hyperfine coupling is small, so that $\omega_{\downarrow / \uparrow, l} \simeq \omega_{I}$. This is confirmed by the more quantitative modeling below.

\subsubsection{Comparison with the model}

As explained above, the measured echo signal results from the contribution of all $18 \mathrm{Bi}: \mathrm{Si}$ transitions because of strain broadening. To model the data, we therefore apply the fictitious spin-1/2 model to each transition, and sum the result- 

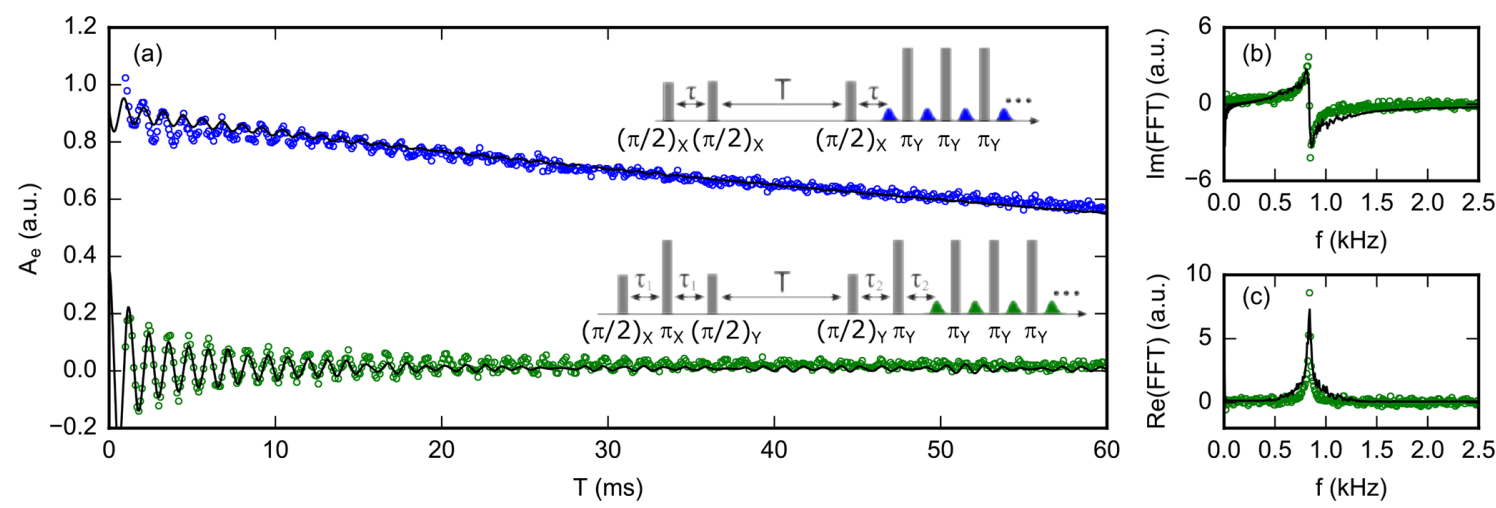

Figure 11. (a) Three-pulse (blue circles) and five-pulse (green circles) ESEEM signals of Bi : Si donors at $B_{0}=0.1 \mathrm{mT}$. Black lines are simulations assuming a ${ }^{29} \mathrm{Si}$ concentration of $p=4.4 \times 10^{-4}$. (b) Imaginary and (c) real part of the Fourier transform of the five-pulse ESEEM data. The spectrum only contains a peak at $850 \mathrm{~Hz}$, which is the ${ }^{29} \mathrm{Si}$ nuclei Larmor frequency at this field.

ing echo amplitudes weighted by their relative contribution, which we determine using numerical simulations described in the Supplement.

Moreover, as discussed in Sect. 3.2, and in contrast to the erbium case, the fictitious spin model for a given transition needs to be validated in the low- $B_{0}$ regime because the energy difference between neighboring hyperfine levels of the bismuth donor manifold $\left(E_{m}^{ \pm}-E_{m-1}^{ \pm}\right) / h \simeq 0.3 \mathrm{MHz}$ for $B_{0}=0.1 \mathrm{mT}$ is comparable to or even lower than the hyperfine coupling to some ${ }^{29} \mathrm{Si}$ nuclei. In that case, the hyperfine interaction induces significant mixing between the bismuth donor and the ${ }^{29} \mathrm{Si}$ eigenstates, and we should describe the coupled "electron spin $\mathbf{S}_{0}+{ }^{209} \mathrm{Bi}$ nuclear spin $\mathbf{I}_{0}+{ }^{29} \mathrm{Si} \mathrm{nu}-$ clear spin I" system as a single 40-level quantum system.

This study is described in Sect. S5 for a ${ }^{29} \mathrm{Si}$ with strong hyperfine coupling $(\geq 200 \mathrm{kHz})$. The state mixing makes many transitions EPR allowed, and the interference between these transitions causes fast oscillations in the spin-echo signal, as seen in Fig. S7 in the Supplement. The frequencies of these oscillations depend greatly on the local Overhauser field on the donor electron spin. Since the latter has a large inhomogeneous broadening $(\sim 0.5 \mathrm{MHz})$, the ensemble average leads to a rapid decay of the signal $(<1 \mu \mathrm{s})$. Given the ${ }^{29} \mathrm{Si}$ concentration, about $10 \%$ of the donors have one or more ${ }^{29} \mathrm{Si}$ with coupling $>300 \mathrm{kHz}$ in the proximity, which therefore leads to a rapid decay of the total echo signal within $\sim 1 \mu$ s by about $10 \%$. In the experimental data, this fast decay is not visible because the echo signal is measured at longer times, and therefore the ESEEM signals presented in Fig. S5 are those from ${ }^{29} \mathrm{Si}$ with couplings $<200 \mathrm{kHz}$.

As for spins with a coupling strength between 20 and $200 \mathrm{kHz}$, they lead to ESEEM amplitude much less than $1 \%$, as shown in Figs. S7-S9. For nuclear spins with a hyperfine coupling $<100 \mathrm{kHz}$, the fictitious spin model produces results with negligible errors of the modulation frequencies from the exact solution (Figs. S5 and S6). Furthermore, the systematic numerical studies (Figs. S9) show that a nearby
Si nuclear spin with coupling $<100 \mathrm{kHz}$ has little effect on the ESEEM due to other distant nuclear spins.

Considering these different contributions of $\mathrm{Si}$ nuclear spins of different hyperfine couplings, as discussed in the paragraph above and in more details in the Supplement, we apply the fictitious spin-1/2 model to each EPR-allowed transition of the bismuth donor manifold, considering only Si nuclear spins that have a hyperfine coupling weaker than a certain cut-off which we choose as $20 \mathrm{kHz}$, and discarding all the others.

For each transition, we compute the hyperfine parameters that enter the fictitious spin- $1 / 2$ model for all sites of the silicon lattice. We then generate a large number of random configurations of nuclear spins. We compute the corresponding two-, three-, or five-pulse ESEEM signal using the analytical formulas of Sect. 2.4 after discarding all nuclei whose hyperfine coupling is larger than $20 \mathrm{kHz}$. We average the signal for one configuration over all bismuth donor transitions using the weights determined by simulation and then average the results over all the configurations computed. In this way, we obtain the curves shown in Fig. 9.

We use the two-pulse echo dataset to determine the most likely sample concentration in ${ }^{29} \mathrm{Si}$, using $p$ as a fitting parameter. As seen in Fig. 9b, the best fit is obtained for $p=4.4 \pm 1 \times 10^{-4}$, which is compatible with the specified $5 \times 10^{-4}$. The agreement is satisfactory but not perfect, as seen, for instance, in the amplitude of the short-time ESEEM oscillations which are lower in the measurements than in the simulations, particularly at larger field. Also, the peak at $2 \omega_{I}$ is notably broader and has a lower amplitude than in the experiment.

For the fitted value of $p$, the three- and five-pulse theoretical signals are also computed and found to be in overall agreement with the data, even though the decay of the ESEEM signal predicted by the model is faster than in the experiment and correspondingly the predicted ESEEM spectrum broader than the data. 


\section{Discussion and conclusion}

We have reported two-, three-, and five-pulse ESEEM measurements using a quantum-limited EPR spectrometer on two model systems: erbium ions in $\mathrm{CaWO}_{4}$ matrix and bismuth donors in silicon. Whereas the erbium measurements are done in a commonly used regime of high field, the bismuth donor measurements are performed in an unusual regime of low nuclear-spin density, low hyperfine coupling, and almost zero magnetic field. Good agreement is found with the simplest analytical ESEEM models.

Having demonstrated that ESEEM is feasible in a millikelvin quantum-limited EPR spectrometer setup on two model spin systems, it is worth speculating in broader terms about its potential for real-world hyperfine spectroscopy. First, high magnetic fields are desirable for a better spectral resolution. Superconducting resonators in $\mathrm{Nb}$, niobium nitride $(\mathrm{NbN})$, or niobium titanium nitride $(\mathrm{NbTiN})$ can retain a high quality factor up to $\sim 1 \mathrm{~T}$ (Graaf et al., 2012; Samkharadze et al., 2016; Mahashabde et al., 2020), so that quantum-limited EPR spectroscopy in the Q band can in principle be envisioned. Resonator bandwidths larger than demonstrated here are also desirable. Given, increasing $\kappa$ in the Purcell regime leads to longer relaxation times $T_{1}$, this should be done with care. One option is to increase also the coupling constant $g$ by further reduction of the resonator mode volume (Ranjan et al., 2020b). Interestingly, this provides another motivation to apply higher magnetic fields, since $g$ is proportional to $\omega_{\mathrm{r}}$. Overall, a resonator at $\omega_{\mathrm{r}} / 2 \pi \simeq 30 \mathrm{GHz}$, in a magnetic field $B_{0} \simeq 1 \mathrm{~T}$, and with a $\kappa / 2 \pi \sim 10 \mathrm{MHz}$ bandwidth seems within reach, while keeping the Purcell $T_{1}$ well below $1 \mathrm{~s}$. Such a high-bandwidth, high-sensitivity EPR spectrometer would be ideally suited for studying surface defects. One potential concern, however, is the power-handling capability of the resonator, as the kinetic inductance causes a non-linear response at high power.

Code and data availability. All code and data necessary for generating Figs. 6-11 can be found at https://doi.org/10.7910/DVN/ZJ2EEX (Probst et al., 2020). The analysis and plotting code is written in Python (.py) and Igor (.pxp). These files are sorted according to figure number, with the relevant files for each figure compressed into a single 7zip file (.7z).

Supplement. The supplement related to this article is available online at: https://doi.org/10.5194/mr-1-315-2020-supplement.

Author contributions. SP, MR, MLD, AD, and PB planned and designed the experiment. $\mathrm{ZZ}$ and $\mathrm{PG}$ prepared the $\mathrm{Er}: \mathrm{CaWO}_{4}$ crystal. JM prepared and provided the bismuth-donor-implanted silicon sample. SP, MR, and MLD fabricated the devices, set up the experiment, and acquired the data. SP, GLZ, MR, VR, MLD, BA, AD, RBL, TC, PG, and PB worked on the data analysis. The project was supervised by RBL and PB. All authors contributed to manuscript preparation.

Competing interests. The authors declare that they have no conflict of interest.

Acknowledgements. We thank Pascal Sénat, Dominique Duet, and Jean-Claude Tack for the technical support and are grateful for fruitful discussions within the Quantronics group. We acknowledge IARPA and Lincoln Labs for providing a Josephson traveling-wave parametric amplifier used in some of the measurements. We acknowledge support of the European Research Council under the European Community's Seventh Framework Programme (FP7/20072013) and under the European Union's Horizon 2020 research and innovation programme of the Agence Nationale de la Recherche under the Chaire Industrielle NASNIQ supported by Atos, the QIPSE project (Hong Kong RGC - French ANR Joint Scheme Fund Project A-CUHK403/15), and the MIRESPIN project, and of Région Îlede-France Domaine d'Interet Majeur SIRTEQ under grant REIMIC. Miloš Rančić acknowledges a Marie Curie Individual Grant of the European Union. Andrin Doll acknowledges a SNSF mobility fellowship.

Financial support. This research has been supported by the European Commission through the H2020 Marie SkłodowskaCurie Action (SMERC (grant no. 792727)), the Seventh Framework Programme (FP7/2007-2013) (CIRQUSS (grant agreement no. 615767)), and the Horizon 2020 Framework Programme, H2020 Excellent Science (LOQO-MOTIONS (grant agreement no. 771493)); the Agence Nationale de la Recherche (grants nos. ANR17-CHIN-0001 and A-CUHK403/15); the Domaine d'Intérêt Majeur Science et Ingénierie en Région Île-de-France pour les Technologies (SIRTEQ (grant no. REIMIC)), and the Swiss National Science Foundation (SNSF) (grant no. 177732).

Review statement. This paper was edited by Robert Bittl and reviewed by Graham Smith and Edgar Groenen.

\section{References}

Abe, E., Tyryshkin, A. M., Tojo, S., Morton, J. J. L., Witzel, W. M., Fujimoto, A., Ager, J. W., Haller, E. E., Isoya, J., Lyon, S. A., Thewalt, M. L. W., and Itoh, K. M.: Electron spin coherence of phosphorus donors in silicon: Effect of environmental nuclei, Phys. Rev. B, 82, 121201, https://doi.org/10.1103/PhysRevB.82.121201, 2010.

Abragam, A. and Bleaney, B.: Electron Paramagnetic Resonance of Transition Ions, OUP Oxford, UK, google-Books-ID: ASNoAgAAQBAJ, ISBN 987-0-19-965152-8, 13 pp., 2012.

Antipin, A., Katyshev, A., Kurkin, I., and Shekun, L.: Paramagnetic resonance and spin-lattice relaxation of Er3+ and Tb3+ ions in CaWO4 crystal lattice, Sov. Phys. Solid State, 10, 468-474, 1968. 
Artzi, Y., Twig, Y., and Blank, A.: Induction-detection electron spin resonance with spin sensitivity of a few tens of spins, Appl. Phys. Lett., 106, 084104, https://doi.org/10.1063/1.4913806, 2015.

Baumann, S., Paul, W., Choi, T., Lutz, C. P., Ardavan, A., and Heinrich, A. J.: Electron paramagnetic resonance of individual atoms on a surface, Science, 350, 417-420, https://doi.org/10.1126/science.aac8703, 2015.

Benningshof, O. W. B., Mohebbi, H. R., Taminiau, I. A. J., Miao, G. X., and Cory, D. G.: Superconducting microstrip resonator for pulsed ESR of thin films, J. Magn. Reson., 230, 84-87, https://doi.org/10.1016/j.jmr.2013.01.010, 2013.

Bienfait, A., Pla, J., Kubo, Y., Stern, M., Zhou, X., Lo, C.-C., Weis, C., Schenkel, T., Thewalt, M., Vion, D., Esteve, D., Julsgaard, B., Moelmer, K., Morton, J., and Bertet, P.: Reaching the quantum limit of sensitivity in electron spin resonance, Nat. Nanotechnol., 11, 253-257, https://doi.org/10.1038/nnano.2015.282, 2015.

Bienfait, A., Pla, J., Kubo, Y., Zhou, X., Stern, M., Lo, C.-C., Weis, C., Schenkel, T., Vion, D., Esteve, D., Morton, J., and Bertet, P.: Controlling Spin Relaxation with a Cavity, Nature, 531, 74-77, https://doi.org/10.1038/nature16944, 2016.

Byrd, R., Lu, P., Nocedal, J., and Zhu, C.: A Limited Memory Algorithm for Bound Constrained Optimization, SIAM J. Sci. Comput., 16, 1190-1208, https://doi.org/10.1137/0916069, 1995.

Car, B., Veissier, L., Louchet-Chauvet, A., Le Gouët, J.L., and Chanelière, T.: Selective Optical Addressing of Nuclear Spins through Superhyperfine Interaction in Rare-Earth Doped Solids, Phys. Rev. Lett., 120, 197401, https://doi.org/10.1103/PhysRevLett.120.197401, 2018.

Childress, L., Dutt, M. V. G., Taylor, J. M., Zibrov, A. S., Jelezko, F., Wrachtrup, J., Hemmer, P. R., and Lukin, M. D.: Coherent Dynamics of Coupled Electron and Nuclear Spin Qubits in Diamond, Science, 314, 281-285, https://doi.org/10.1126/science.1131871, 2006.

Day, P. K., LeDuc, H. G., Mazin, B. A., Vayonakis, A., and Zmuidzinas, J.: A broadband superconducting detector suitable for use in large arrays, Nature, 425, 817-821, https://doi.org/10.1038/nature02037, 2003.

Eichler, C., Sigillito, A. J., Lyon, S. A., and Petta, J. R.: Electron Spin Resonance at the Level of $10^{4}$ Spins Using Low Impedance Superconducting Resonators, Phys. Rev. Lett., 118, 037701, https://doi.org/10.1103/PhysRevLett.118.037701, 2017.

Elzerman, J. M., Hanson, R., Beveren, L. H. W. v., Witkamp, B., Vandersypen, L. M. K., and Kouwenhoven, L. P.: Single-shot read-out of an individual electron spin in a quantum dot, Nature, 430, 431-435, https://doi.org/10.1038/nature02693, 2004.

Feher, G.: Electron Spin Resonance Experiments on Donors in Silicon. I. Electronic Structure of Donors by the Electron Nuclear Double Resonance Technique, Phys. Rev., 114, 1219-1244, https://doi.org/10.1103/PhysRev.114.1219, 1959.

Graaf, S. E. d., Danilov, A. V., Adamyan, A., Bauch, T., and Kubatkin, S. E.: Magnetic field resilient superconducting fractal resonators for coupling to free spins, J. Appl. Phys., 112, 123905, https://doi.org/10.1063/1.4769208, 2012.

Guillot-Noël, O., Vezin, H., Goldner, P., Beaudoux, F., Vincent, J., Lejay, J., and Lorgeré, I.: Direct observation of rare-earthhost interactions in $\mathrm{Er}: \mathrm{Y}_{2} \mathrm{SiO}_{5}$, Phys. Rev. B, 76, 180408, https://doi.org/10.1103/PhysRevB.76.180408, 2007.

Hale, E. B. and Mieher, R. L.: Shallow Donor Electrons in Silicon. II. Considerations Regarding the
Fermi Contact Interactions, Phys. Rev., 184, 751-759, https://doi.org/10.1103/physrev.184.751, 1969.

Jelezko, F., Gaebel, T., Popa, I., Gruber, A., and Wrachtrup, J.: Observation of Coherent Oscillations in a Single Electron Spin, Phys. Rev. Lett., 92, 076401, https://doi.org/10.1103/PhysRevLett.92.076401, 2004.

Kasumaj, B. and Stoll, S.: 5- and 6-pulse electron spin echo envelope modulation (ESEEM) of multinuclear spin systems, J. Magn. Reson., 190, 233-247, https://doi.org/10.1016/j.jmr.2007.11.001, 2008.

Kohn, W. and Luttinger, J. M.: Theory of Donor States in Silicon, Physical Review, 98, 915-922, https://doi.org/10.1103/PhysRev.98.915, 1955.

Kubo, Y., Ong, F. R., Bertet, P., Vion, D., Jacques, V., Zheng, D., Dréau, A., Roch, J.-F., Auffeves, A., Jelezko, F., Wrachtrup, J., Barthe, M. F., Bergonzo, P., and Esteve, D.: Strong Coupling of a Spin Ensemble to a Superconducting Resonator, Phys. Rev. Lett., 105, 140502, https://doi.org/10.1103/PhysRevLett.105.140502, 2010.

Macklin, C., O’Brien, K., Hover, D., Schwartz, M. E., Bolkhovsky, V., Zhang, X., Oliver, W. D., and Siddiqi, I.: A near-quantumlimited Josephson traveling-wave parametric amplifier, Science, 350, 307-310, https://doi.org/10.1126/science.aaa8525, 2015.

Mahashabde, S., Otto, E., Montemurro, D., de Graaf, S., Kubatkin, S., and Danilov, A.: Fast Tunable High- $\boldsymbol{Q}$-Factor Superconducting Microwave Resonators, Phys. Rev. Applied 14, 044040, https://doi.org/10.1103/PhysRevApplied.14.044040, 2020.

Mansir, J., Conti, P., Zeng, Z., Pla, J., Bertet, P., Swift, M., Van de Walle, C., Thewalt, M., Sklenard, B., Niquet, Y., and Morton, J.: Linear Hyperfine Tuning of Donor Spins in Silicon Using Hydrostatic Strain, Phys. Rev. Lett., 120, 167701, https://doi.org/10.1103/PhysRevLett.120.167701, 2018

Mims, W. B.: Envelope Modulation in SpinEcho Experiments, Phys. Rev. B, 5, 2409-2419, https://doi.org/10.1103/physrevb.5.2409, 1972.

Mims, W. B., Nassau, K., and McGee, J. D.: Spectral Diffusion in Electron Resonance Lines, Phys. Rev., 123, 2059-2069, https://doi.org/10.1103/PhysRev.123.2059, 1961.

Mims, W. B., Davis, J. L., and Peisach, J.: The exchange of hydrogen ions and of water molecules near the active site of cytochrome $c$, J. Magn. Reson., 86, 273-292, https://doi.org/10.1016/0022-2364(90)90260-G, 1990.

Mohammady, M. H., Morley, G. W., and Monteiro, T. S.: Bismuth Qubits in Silicon: The Role of EPR Cancellation Resonances, Phys. Rev. Lett., 105, 067602, https://doi.org/10.1103/physrevlett.105.067602, 2010.

Morello, A., Pla, J. J., Zwanenburg, F. A., Chan, K. W., Tan, K. Y., Huebl, H., Mottonen, M., Nugroho, C. D., Yang, C., van Donkelaar, J. A., Alves, A. D. C., Jamieson, D. N., Escott, C. C., Hollenberg, L. C. L., Clark, R. G., and Dzurak, A. S.: Single-shot readout of an electron spin in silicon, Nature, 467, 687-691, https://doi.org/10.1038/nature09392, 2010.

Narkowicz, R., Suter, D., and Niemeyer, I.: Scaling of sensitivity and efficiency in planar microresonators for electron spin resonance, Review of Scientific Instruments, 79, 084702, https://doi.org/10.1063/1.2964926, 2008.

Pla, J., Bienfait, A., Pica, G., Mansir, J., Mohiyaddin, F., Zeng, Z., Niquet, Y., Morello, A., Schenkel, T., Morton, J., and Bertet, P.: Strain-Induced Spin-Resonance 
Shifts in Silicon Devices, Phys. Rev. Appl., 9, 044014, https://doi.org/10.1103/PhysRevApplied.9.044014, 2018.

Pla, J. J., Tan, K. Y., Dehollain, J. P., Lim, W. H., Morton, J. J. L., Jamieson, D. N., Dzurak, A. S., and Morello, A.: A single-atom electron spin qubit in silicon, Nature, 489, 541-545, https://doi.org/10.1038/nature11449, 2012.

Probst, S., Rotzinger, H., Wünsch, S., Jung, P., Jerger, M., Siegel, M., Ustinov, A. V., and Bushev, P. A.: Anisotropic Rare-Earth Spin Ensemble Strongly Coupled to a Superconducting Resonator, Phys. Rev. Lett., 110, 157001, https://doi.org/10.1103/PhysRevLett.110.157001, 2013.

Probst, S., Rotzinger, H., Ustinov, A. V., and Bushev, P. A.: Microwave multimode memory with an erbium spin ensemble, Phys. Rev. B, 92, 014421, https://doi.org/10.1103/PhysRevB.92.014421, 2015.

Probst, S., Bienfait, A., Campagne-Ibarcq, P., Pla, J. J., Albanese, B., Barbosa, J. F. D. S., Schenkel, T., Vion, D., Esteve, D., Moelmer, K., Morton, J. J. L., Heeres, R., and Bertet, P.: Inductive-detection electron-spin resonance spectroscopy with 65 spins $/ \sqrt{\mathrm{Hz}}$ sensitivity, Appl. Phys. Lett., 111, 202604, https://doi.org/10.1063/1.5002540, 2017.

Probst, S., Ranjan, V., Ansel, Q., Heeres, R., Albanese, B., Albertinale, E., Vion, D., Esteve, D., Glaser, S. J., Sugny, D., and Bertet, P.: Shaped pulses for transient compensation in quantum-limited electron spin resonance spectroscopy, J. Magn. Reson., 303, 4247, https://doi.org/10.1016/j.jmr.2019.04.008, 2019.

Probst, S., Zhang, G., Rančić, M., Ranjan, V., Le Dantec, M., Zhang, Z., Albanese, B., Doll, A., Liu, R. B., Morton, J., Chanelière, T., Goldner, P., Vion, D., Esteve, D., and Bertet, P.: Replication Data for: Hyperfine spectroscopy in a quantum-limited spectrometer (2020), Harvard Dataverse, https://doi.org/10.7910/DVN/ZJ2EEX, 2020.

Ranjan, V., Probst, S., Albanese, B., Doll, A., Jacquot, O., Flurin, E., Heeres, R., Vion, D., Esteve, D., Morton, J. J. L., and Bertet, P.: Pulsed electron spin resonance spectroscopy in the Purcell regime, J. Magn. Reson., 310, 106662, https://doi.org/10.1016/j.jmr.2019.106662, 2020a.

Ranjan, V., Probst, S., Albanese, B., Schenkel, T., Vion, D., Esteve, D., Morton, J. J. L., and Bertet, P.: Electron spin resonance spectroscopy with femtoliter detection volume, Appl. Phys. Lett., 116, 184002, https://doi.org/10.1063/5.0004322, 2020b.

Rowan, L. G., Hahn, E. L., and Mims, W. B.: ElectronSpin-Echo Envelope Modulation, Phys. Rev., 137, A61-A71, https://doi.org/10.1103/physrev.137.a61, 1965.

Rugar, D., Budakian, R., Mamin, H., and Chui, B.: Single spin detection by magnetic resonance force microscopy, Nature, 430, 329-332, https://doi.org/10.1038/nature02658, 2004.

Samkharadze, N., Bruno, A., Scarlino, P., Zheng, G., DiVincenzo, D., DiCarlo, L., and Vandersypen, L.: High-KineticInductance Superconducting Nanowire Resonators for Circuit QED in a Magnetic Field, Phys. Rev. Appl., 5, 044004, https://doi.org/10.1103/PhysRevApplied.5.044004, 2016.
Schweiger, A. and Jeschke, G.: Principles of pulse electron paramagnetic resonance, 1st edn., Oxford University Press, Oxford, UK, ISBN: 019850634 1, 2001.

Sidabras, J. W., Duan, J., Winkler, M., Happe, T., Hussein, R., Zouni, A., Suter, D., Schnegg, A., Lubitz, W., and Reijerse, E. J.: Extending electron paramagnetic resonance to nanoliter volume protein single crystals using a self-resonant microhelix, Science Advances, 5, eaay1394, https://doi.org/10.1126/sciadv.aay1394, 2019.

Sigillito, A. J., Malissa, H., Tyryshkin, A. M., Riemann, H., Abrosimov, N. V., Becker, P., Pohl, H.-J., Thewalt, M. L. W., Itoh, K. M., Morton, J. J. L., Houck, A. A., Schuster, D. I., and Lyon, S. A.: Fast, low-power manipulation of spin ensembles in superconducting microresonators, Appl. Phys. Lett., 104, 222407, https://doi.org/10.1063/1.4881613, 2014.

Sigillito, A., Tyryshkin, A., Schenkel, T., Houck, A. A., and Lyon, S. A.: All-electric control of donor nuclear spin qubits in silicon, Nature Nanotech, 12, 958-962, https://doi.org/10.1038/nnano.2017.154, 2017.

Song, L., Liu, Z., Kaur, P., Esquiaqui, J. M., Hunter, R. I., Hill, S., Smith, G. M., and Fanucci, G. E.: Toward increased concentration sensitivity for continuous wave EPR investigations of spinlabeled biological macromolecules at high fields, J. Magn. Reson., 265, 188-196, https://doi.org/10.1016/j.jmr.2016.02.007, 2016.

Veldhorst, M., Hwang, J. C. C., Yang, C. H., Leenstra, A. W., de Ronde, B., Dehollain, J. P., Muhonen, J. T., Hudson, F. E., Itoh, K. M., Morello, A., and Dzurak, A. S.: An addressable quantum dot qubit with fault-tolerant control-fidelity, Nat. Nanotechnol., 9, 981-985, https://doi.org/10.1038/nnano.2014.216, 2014.

Wallace, W. J. and Silsbee, R. H.: Microstrip resonators for electron-spin resonance, Review of Scientific Instruments, 62, 1754 1766, https://doi.org/10.1063/1.1142418, 1991.

Witzel, W. M., Hu, X., and Das Sarma, S.: Decoherence induced by anisotropic hyperfine interaction in Si spin qubits, Phys. Rev. B, 76, 035212, https://doi.org/10.1103/PhysRevB.76.035212, 2007.

Wolfowicz, G., Tyryshkin, A. M., George, R. E., Riemann, H., Abrosimov, N. V., Becker, P., Pohl, H.-J., Thewalt, M. L. W., Lyon, S. A., and Morton, J. J. L.: Atomic clock transitions in silicon-based spin qubits, Nat. Nanotechnol., 8, 561-564, https://doi.org/10.1038/nnano.2013.117, 2013.

Wrachtrup, J., Von Borczyskowski, C., Bernard, J., Orritt, M., and Brown, R.: Optical detection of magnetic resonance in a single molecule, Nature, 363, 244-245, https://doi.org/10.1038/363244a0, 1993.

Zhou, X., Schmitt, V., Bertet, P., Vion, D., Wustmann, W., Shumeiko, V., and Esteve, D.: High-gain weakly nonlinear flux-modulated Josephson parametric amplifier using a SQUID array, Phys. Rev. B, 89, 214517, https://doi.org/10.1103/PhysRevB.89.214517, 2014. 